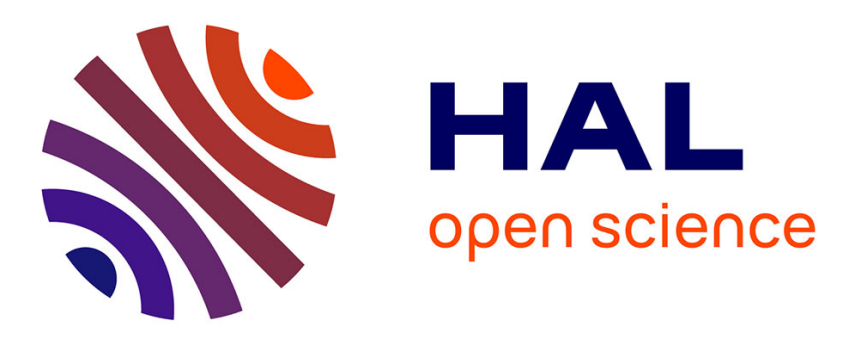

\title{
Electronic Structure of Nanoalloys: A Guide of Useful Concepts and Tools
}

\author{
Guy Treglia, Christine Goyhenex, Christine Mottet, Christine Legrand, \\ François Ducastelle
}

\section{> To cite this version:}

Guy Treglia, Christine Goyhenex, Christine Mottet, Christine Legrand, François Ducastelle. Electronic Structure of Nanoalloys: A Guide of Useful Concepts and Tools. Nanoalloys, pp.159 - 195, 2012, 10.1007/978-1-4471-4014-6_5 . hal-03038203

\section{HAL Id: hal-03038203 https://hal.science/hal-03038203}

Submitted on 3 Dec 2020

HAL is a multi-disciplinary open access archive for the deposit and dissemination of scientific research documents, whether they are published or not. The documents may come from teaching and research institutions in France or abroad, or from public or private research centers.
L'archive ouverte pluridisciplinaire HAL, est destinée au dépôt et à la diffusion de documents scientifiques de niveau recherche, publiés ou non, émanant des établissements d'enseignement et de recherche français ou étrangers, des laboratoires publics ou privés. 


\title{
Electronic Structure of Nanoalloys: A Guide of Useful Concepts and Tools
}

\author{
G. Tréglia, C. Goyhenex, C. Mottet, B. Legrand and F. Ducastelle
}

\begin{abstract}
The aim of this lecture is to give an overlook about methods developed in infinite (bulk) and semi-infinite (surface) metallic materials and some tracks to extend them to finite size systems. In this framework we will first study the effect of bond breaking and dimension lowering on electronic structure, at surfaces of pure metals (surface states, atomic level shifts, reconstructions and relaxations) and in monometallic clusters. Then we will illustrate the influence of chemical ordering on electronic structure (and vice versa) by considering firstly bulk alloys (diagonal versus off-diagonal disorder) and then bimetallic surfaces (stress effect induced by either surface segregation or epitaxial growth). These two approaches will then naturally be combined in the peculiar case of nanoalloys. The methods will be developed following two main goals. The first one is to determine local electronic densities of states (LDOS), the knowledge of which is essential to the understanding and the analysis of nano-objects. The second one is to derive from these LDOS energetic models well suited to both the degree of complexity of the systems under study (bulk and surface crystalline structure, chemical ordering, ...) and their implementation in numerical simulations (Molecular Dynamics, Monte
\end{abstract}

G. Tréglia $(\bowtie) \cdot$ C. Mottet

CINaM - CNRS, Campus de Luminy, Case 913, 13288

Marseille Cedex 9, France

e-mail: treglia@cinam.univ-mrs.fr

C. Goyhenex

IPCMS - CNRS - UDS, 23 rue du Loess, 67034

Strasbourg cedex 2, France

B. Legrand

SRMP - DMN, CEA Saclay, 91191

Gif sur Yvette Cedex, France

F. Ducastelle

LEM - CNRS/ONERA, B.P. 72, 92322

Châtillon cedex, France 
Carlo). The different sections of the lecture will be illustrated by examples issued from studies performed on systems which can be considered as archetypal in the nano-alloy community, such as $\mathrm{CoPt}, \mathrm{CoAu}$ and $\mathrm{CuAg}$.

\section{Introduction}

The peculiar properties of nanoalloys depend on the local electronic structure on the various inequivalent sites resulting from their chemical and morphological structures, which in turn depend on this electronic structure. It is then essential to understand how these structures are coupled to one another and how they vary with the metal species, the concentration and the orientation of the surface for semi-infinite materials and/or the cluster size for finite ones. Modelling these phenomena would indeed allow us to design the best suited binary system for a given property.

The aim of this lecture is to give the tools for characterizing the electronic structure of bimetallic clusters, and to show how they can be used to predict both their atomic and chemical structures. These electronic structure methods extend from ab initio calculations to semi-phenomenological models such as Tight-Binding approximation for transition metals. Since the most commonly used nanoalloys are made of metals of the end of the transition series, we will put some emphasis on the latter by giving some details on moment and continued fraction methods. We will underline how the electronic structure is modified at surface and cluster sites, first for pure metals and then for bimetallic systems. Then, we will show how the energetics of the system (cohesive energy, surface tension, mixing energy) can be derived from electronic structure by using more or less sophisticated many-body potentials (SMA: Second Moment Approximation, TBIM: Tight-Binding Ising Model). This will allow us to get trends as a function of the number of valence electrons for various properties such as the crystalline structure of pure metals, the relaxation or reconstruction of surfaces, the shape of clusters and finally the chemical structure of infinite systems (tendency to ordering or phase separation) and finite ones (surface or site segregation). In turn, we will illustrate the dependence of the local densities of states with respect to the equilibrium (geometrical, chemical) environment defined as above.

\section{Concepts and Methods (Pure Bulk Metal)}

\subsection{Chemical Bonding and Periodic Table}

The Hamiltonian of a system with $\mathrm{N}$ nuclei located at $R$, and $N_{e}$ electrons, located at $r$ with a spin $\sigma$, writes in the most general way: 


$$
H=\sum_{I=1, N} \frac{P_{I}^{2}}{2 M_{I}}+\sum_{i=1, N_{e}} \frac{p_{i}^{2}}{2 m}+\sum_{i, j} \frac{e^{2}}{\left|\mathbf{r}_{i}-\mathbf{r}_{j}\right|}+\sum_{I, J} \frac{Z_{I} Z_{J} e^{2}}{\left|\mathbf{R}_{i}-\mathbf{R}_{j}\right|}-\sum_{i, I} \frac{Z_{I} e^{2}}{\left|\mathbf{r}_{i}-\mathbf{R}_{j}\right|}
$$

This Hamiltonian acts on a many-body wave function $\Phi(x, R)$ which depends on both nuclei $(R)$ and electron $(x \cong(r, \sigma))$ coordinates. Due to the mass difference between the electrons and nuclei, we can decouple their respective movements (adiabatic approximation) which allows one to write the total wave-function as the product of those of the electrons $\psi(x, R)$ and of the nuclei $\chi(R)$ : $\Phi(x, R)=\psi(x, R) \cdot \chi(R)$. Solving exactly the Schrödinger equation $H \psi=E \psi$ for the electrons is only possible in the simple case of the hydrogen atom with only one proton and one electron. In that case, using spherical coordinates, the solution writes as the product of a radial function and of a spherical harmonic:

$$
\psi_{n l m}(r, \theta, \varphi)=R_{n}^{l}(r) Y_{l}^{m}(\theta, \phi)
$$

which involves three quantum numbers $n$ (principal: $n \geq 0$ ), $l$ (azimuthal: $n \geq l \geq 0$ ) and $m$ (magnetic: $l \geq m \geq-l$ ), plus a fourth number for the spin $(s= \pm 1 / 2)$. The energy associated to the function $\psi_{n l m}$ only depends on $n\left(E_{n}=-E_{0} / n^{2}\right.$, with $\left.E_{0}=13.6 \mathrm{eV}\right)$ so that the ground state of the system, which corresponds to the minimal energy, is obtained by filling the respective levels as a function of increasing $n$. All the electronic states corresponding to the same energy are then labelled by $n$, even though their properties essentially depend on the value of $l$, which drives the shape of the orbitals (see Fig. 1a), giving rise to the usual denomination: $n s(l=0), n p(l=1), n d(l=2)$, nf $(l=3), \ldots$ The magnetic number gives the degeneracy of each state (i.e. the maximal number of electrons it can contain) which, counting the spin, is: $n s^{2}, n p^{6}, n d^{10}, n f^{14}, \ldots$.

In fact, the degeneracy of the different $l$-levels corresponding to a given $n$ state will then be lifted by introducing interaction between electrons for atoms containing more than one electron, leading to the variation of $E_{n l}$ schematized in Fig. 1b, which directly leads to the classification of all elements within the Mendeleiev classification table. The various types of elements, characterized by the nature of their valence electrons ( $s$ for simple metals, $s p$ for covalent elements, $d$ for transition metals and $s d$ for noble metals) are illustrated in Fig. 2.

\subsection{One electron approximation: band structure (Hartree-Fock, DFT)}

In condensed matter, one has to deal with the general problem of $N_{e}$ electrons moving in the potential $V^{\text {ion }}$ of $N$ fixed ions. The Hamiltonian then writes:

$$
H=\sum_{i=1}^{N_{e}}\left(\frac{p_{i}^{2}}{2 m}+V^{i o n}\left(\mathbf{r}_{i}\right)\right)+\frac{1}{2} \sum_{i, j=1}^{N_{e}} \frac{e^{2}}{\left|r_{i}-r_{j}\right|}
$$


(a)

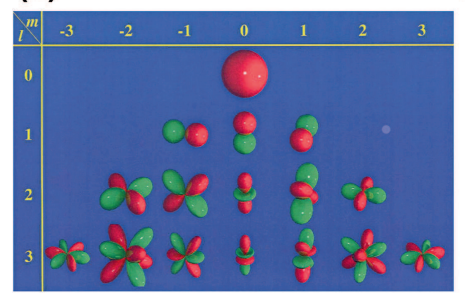

(b)

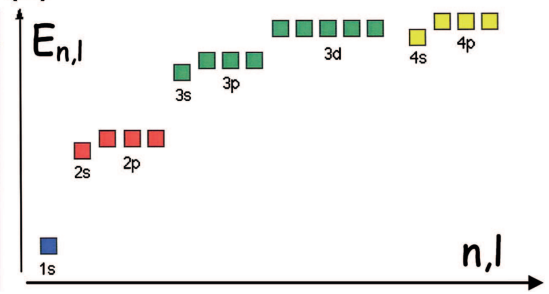

Fig. 1 Schematic orbitals (left) for the various $(l, m)$ numbers and corresponding level energies (right)

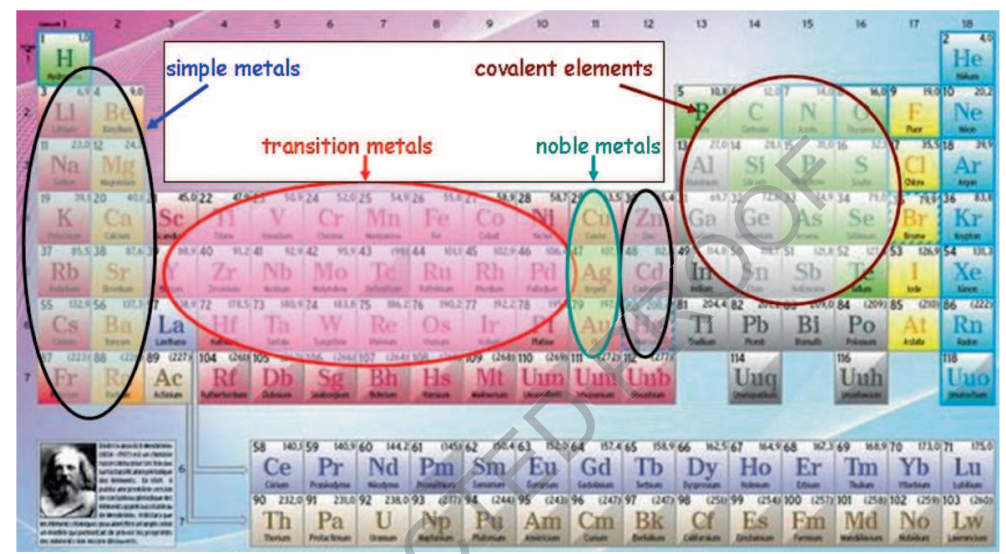

Fig. 2 Mendeleïev periodic classification table

The motions of the electrons are then correlated due to their Coulomb interactions, which is a quantic many-body problem involving at least all valence electrons (external shells). Assuming that a given (single) electron interacts with all the others by means of an effective mean-field $V^{\text {eff }}(r)$, Eq. (3) reduces to a "one electron" Hamiltonian, the eigenfunctions and eigenvalues of which are solutions of the Schrödinger equation:

$$
\left[\frac{p^{2}}{2 m}+V^{i o n}(\mathbf{r})+V^{e f f}(\mathbf{r})\right] \psi_{\alpha}(\mathbf{r})=\varepsilon_{\alpha} \psi_{\alpha}(\mathbf{r})
$$

The ground state of the system at $T=0 \mathrm{~K}$ is obtained by stacking the electrons in the lowest energy states available, leading to $N$-body states characterized by the occupation numbers (or Fermi functions) $f_{\alpha}$ which are defined such as $f_{\alpha}=1$ if $\varepsilon_{\alpha}<E_{F}$ ( $E_{F}$ being the Fermi level) and $f_{\alpha}=0$ otherwise. The spatial density of states is then defined as:

$$
n(\mathbf{r})=\sum_{\alpha} f_{\alpha}\left|\psi_{\alpha}(\mathbf{r})\right|^{2}
$$


Within the Hartree approximation, the effective potential writes:

$$
V^{e f f}(\mathbf{r})=V^{H}(\mathbf{r})=e^{2} \int d \vec{r}^{\prime} \frac{n\left(\mathbf{r}^{\prime}\right)}{\left|\mathbf{r}-\mathbf{r}^{\prime}\right|}
$$

The Eq. (4) is solved by an iterative procedure, starting from an initial (guessed) density of states $\mathrm{n}(\mathrm{r})$, which allows one to calculate $V^{\text {eff }}(r)$ using (6), then to solve the Eq. (4) from which one obtains $\psi_{\alpha}$ and then $n(r)$ through (5). The procedure is then iterated as long as self-consistency is not achieved.

Unfortunately, in spite of its physical content, this remains an approximation which does not account for the correlated motion of all the electrons. In particular, this Hartree approximation does not account for the Pauli principle and then totally misses the existence of the so-called exchange and correlation hole which makes electrons avoiding each other at short distance. This is somewhat corrected in the Hartree-Fock approximation which improves the Hartree potential by including a so-called exchange contribution, which damps the Coulomb potential contribution for parallel spins. Unfortunately, this is a rather asymmetric way to treat the electron interactions since all the electrons should avoid one another. Therefore, whereas the electronic correlations are completely neglected in the Hartree scheme, they are treated in a too much asymmetric way in the Hartree-Fock approximation.

A main progress with the Density Functional Theory (DFT), which is the most widely used ab initio method, is that it treats the correlations in a more symmetric way. It is based on the Hohenberg and Kohn theorem [1], which assumes that the ground state energy $E_{O}$ of an inhomogeneous interacting electron gas under an external potential $V^{i o n}$ can be written as a functional of the charge density $n(r)$, $E_{0}=E_{0}[n(r)]$, which is minimum for the real density of the system. This leads to write $n(r)$ under the same "one electron" form as (5), using wave functions $\psi \alpha$ which are solutions of a Hamiltonian similar to (4), but with now an effective potential:

$$
V^{e f f}(\mathbf{r})=V^{H}(\mathbf{r})+V^{x c}(\mathbf{r})
$$

which differs from (6) by the introduction of an exchange-correlation term $V^{x c}$, which is the functional derivative of a contribution $E^{x c}[n(r)]$ to $E_{0}[n(r)]$ :

$$
V^{x c}(\mathbf{r})=\frac{\partial E^{x c}[n(\mathbf{r})]}{\partial n(\mathbf{r})}
$$

All the difficulties are then transferred in this term which has to be approximated. Within the usual Local Density Approximation (LDA), one assumes that $E^{x c}$ is a local functional of $n(r)$, i.e., that it is defined from the knowledge of the density at $\mathbf{r}$ only.

$$
E^{x c}[n(\mathbf{r})] \approx \int n(\mathbf{r}) \varepsilon^{x c}[n(\mathbf{r})] d \mathbf{r}
$$




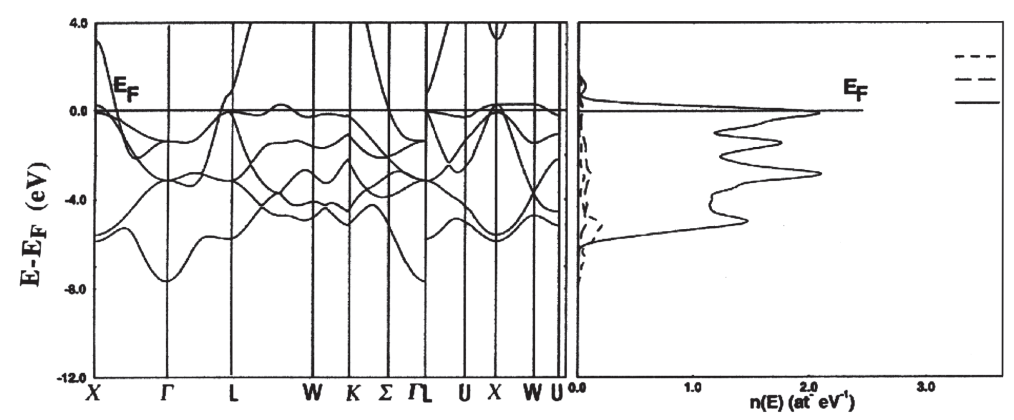

Fig. 3 LMTO calculation of the band structure and density of states of Pd. The full line indicates the $d$-partial LDOS and the dotted and dashed ones the $s-p$ ones. Courtesy of S. Sawaya

$\varepsilon^{x c}(n)$ is the so-called "exchange and correlation" energy of a uniform electron system with density $\mathrm{n}$, which is widely taken as the average value of the exchange potential for a free electron system $\left(\propto n^{1 / 3}\right)$, weighted by an empirical factor $\alpha\left(X_{\alpha}\right.$ method). When this local approximation fails, due to rapid variations of the density, it can be corrected by introducing corrections linked to the gradient of the density: $\vec{\nabla} n(\vec{r})$ (GGA: Generalized Gradient Approximation). Finally, inserting Eqs. (7) an (8) in the Eq. (4) leads to the well-known Kohn-Sham equations which have to be solved iteratively by using the same self-consistent procedure as already described. From the resulting eigenvalues $\varepsilon_{x}$ one can then access to the electronic band structure, and from that to the density of states following:

$$
n(E)=\sum_{\alpha} \delta\left(E-\varepsilon_{\alpha}\right)=\operatorname{Tr} \delta(E-H)
$$

where the operator $\delta(E-H)$ is defined by: $\delta(E-H) \cdot \psi_{\alpha}=\delta\left(E-\varepsilon_{\alpha}\right) . \psi_{\alpha}$ and the trace (Tr) is performed on the electronic states $\alpha$. This is illustrated in the case of Pd (Fig. 3).

Even though DFT is a non parameterized method, it requires performing some important choices, in addition to that of the exchange-correlation term (LDA, GGA). The first one is that of the electron potential among a wide variety: full potential (FP), "muffin tin" (MT) potentials (the potential is calculated exactly in spherical regions centred on the nuclei whereas it is taken equal to zero in the interstitial region), atomic sphere approximation (ASA) or pseudopotentials (PP). The latter have been developed to explain how a nearly-free behaviour of electrons could be consistent with a potential $V^{\text {ion }}$ which diverges in the ion vicinity. Indeed in this region, their wave functions oscillate rapidly to orthogonalize to the inner shell states, leading to a large kinetic energy which almost compensates the potential energy. One can then define weak pseudopotentials associated to pseudonearly free wave functions (e.g. Ashcroft empty core [2]). The second choice is that of the basis which determines the efficiency of the method depending on the system under study. In this framework the plane waves basis provides the 
simplicity and speed of Fourier development whereas localised orbitals (Gaussians for chemists, or numerical in SIESTA) have the advantage to give a quasi-atomic view (consistent with Tight-Binding approximation, see later). Finally augmented methods (APW) combine the best of these two opposite points of view, by calculating wave functions at fixed energy inside an atomic sphere, which are matched to plane waves outside.

The DFT method not only gives very good results concerning both the band structure and density of states, but also for the lattice parameter, elastic constants and cohesive energies, at least when gradient corrections are taken into account. This is very satisfying since this method is ab initio, i.e., "without parameters" (contrary to more empirical methods which will be developed later), which does not mean that it is "without approximation" as shown above! Nevertheless, this method remains less suited for non periodic systems, in presence of defects, and tedious to use coupled with numerical simulations such as Molecular Dynamics... even though Car-Parinello type methods [3] have been developed which take into account simultaneously the movements of ions and electrons. But such methods remain heavy to handle for large systems, which justify developing simpler methods, using semi-empirical potentials suited to the system under study.

\subsection{Tight-Binding Approximation and Local Density of States}

The Tight-Binding (TB) method [4] starts from isolated atoms with discrete levels, which form energy bands when the atomic wave functions overlap... but not too much! It assumes that any one electron electronic state $\psi(r)$, delocalised in the solid, can be written as a linear combination of atomic orbitals (LCAO) $|n, \lambda\rangle$ where $\lambda$ labels the orbital at site $n: \psi(r)=\sum_{n, \lambda} a_{n}^{\lambda}|n, \lambda\rangle$, which is the more justified as the overlap between the orbitals is weak ( $d$ states of transition metals, $s p$ valence electrons of semi-conductors,...). The corresponding TB Hamiltonian then writes:

$$
H=\sum_{n, \lambda}|n, \lambda\rangle\left(\varepsilon_{\lambda, 0}+\alpha_{\lambda}\right)\left\langle n, \lambda\left|+\sum_{n, m, \lambda, \mu}\right| n, \lambda\right\rangle \beta_{n m}^{\lambda \mu}\langle m, \mu|
$$

in which $\varepsilon_{\lambda, 0}, \alpha_{\lambda}$ and $\beta_{n m}^{\lambda \mu}$ are respectively the atomic level, crystal field and hopping integrals, the latter being rapidly damped (after 1st or 2 nd neighbours) and directly related to the bandwidth. Due to the spherical symmetry of atomic potentials, the $[\beta]$ matrix is diagonal for each $l$-value in the basis of spherical harmonics with the $z$-axis along $(m-n)$, with eigenvalues defined as the integrals $\sigma$, $\pi, \delta$ according to the quantum magnetic number $|m|=0,1,2$. This leads to different hopping integrals labelled $s s \sigma, p p \sigma, p p \pi, d d \sigma, d d \pi, d d \delta$ (see Fig. 4) to which are added integrals coupling two different $l: s p \sigma, s d \sigma, p d \sigma, p d \pi$. In this framework, one can define $d-d$ canonical parameters such as: $|d d \sigma| \approx 2|d d \pi|$, $d d \delta \approx 0$ [5]. 


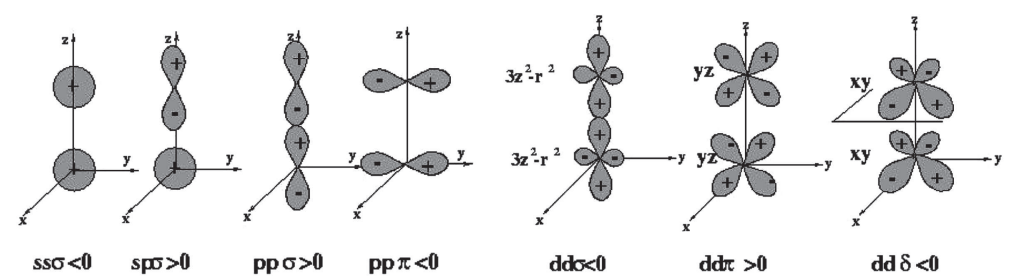

Fig. 4 Schematic $s p-s p$ and $d-d$ hopping integrals

The essential advantage of the method is that it allows working in the direct space to calculate densities and energies, without resorting to diagonalisation of the Hamiltonian, and then without need for the Bloch theorem. This allows one to deal with non crystalline solids and defects. Indeed, one can derive $n(E)$ from the trace of $\delta(E-H)$ Eq. (10) which can be calculated within any basis, and in particular in the basis of atomic orbitals $|n, \lambda\rangle$. More precisely, using mathematical properties of $\delta$-functions, one can define in a simple way the local density of states (LDOS) at a given site $n_{0}$,

$$
n_{n_{0}}(E)=\lim _{\eta \rightarrow 0^{+}}\left[-\frac{\operatorname{Im}}{\pi} \sum_{\lambda}\left\langle n_{0}, \lambda|G(E+i \eta)| n_{0}, \lambda\right\rangle\right]
$$

without resorting to any periodicity condition $\left(n_{0}\right.$ can be a defect site), from the projection of the Green function: $G(z)=(z-H)^{-1}$ on the atomic orbital at site $n_{0}$. This projection writes as a continued fraction [6],

$$
\left\langle n_{0}, \lambda|G(z)| n_{0}, \lambda\right\rangle=\frac{1}{z-a_{1}-\frac{b_{1}^{2}}{z-a_{2}-\frac{b_{2}^{2}}{z-a_{3}-\frac{b_{3}^{2}}{\cdots \cdots}}}}
$$

the coefficients of which can be calculated by two different ways. The first one is to derive them from the knowledge of the $p$ first moments $\mu_{p}$ of $n_{n_{0}}(E)$ :

$$
\begin{aligned}
\mu_{p}\left(n_{0}\right) & =\int_{-\infty}^{+\infty} E^{p} n_{n_{0}}(E) d E=\sum_{\lambda}\left\langle n_{0}, \lambda\left|H^{p}\right| n_{0}, \lambda\right\rangle \\
& =\sum_{\lambda, i \mu j, j, \ldots}\left\langle n_{0}, \lambda|H| i, \mu\right\rangle\langle i, \mu|H| j, v\rangle \ldots\left\langle., .|H| n_{0}, \lambda\right\rangle
\end{aligned}
$$

which gives more and more details on the LDOS when $p$ increases, and are obtained by counting closed paths on the lattice [6]. The second way is to calculate them directly by constructing a new basis tridiagonalising $H$ within the so-called recursion method [7]. The LDOS is the most precise as the number of calculated coefficients is large, since $N$ pairs of exact coefficients ensure the LDOS to have $2 \mathrm{~N}$ exact moments. The problem is then to terminate the continued fraction. For a 


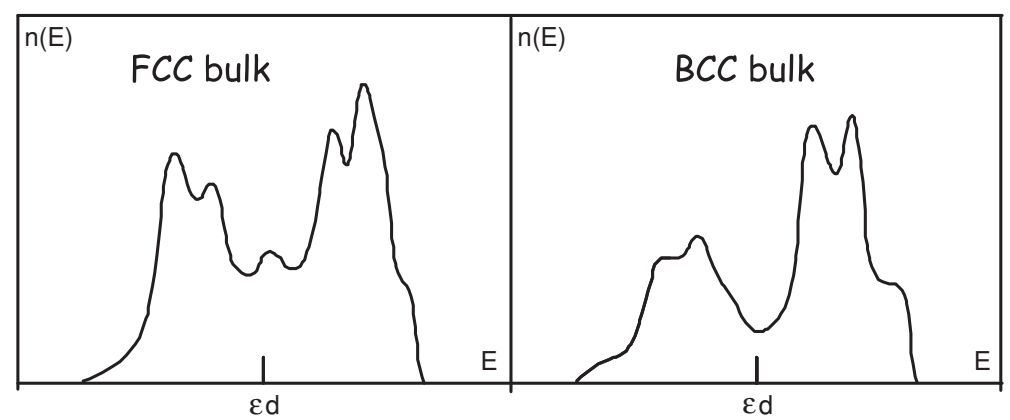

Fig. 5 Typical $d$-LDOS for fcc (left) and bcc (right) bulk structures

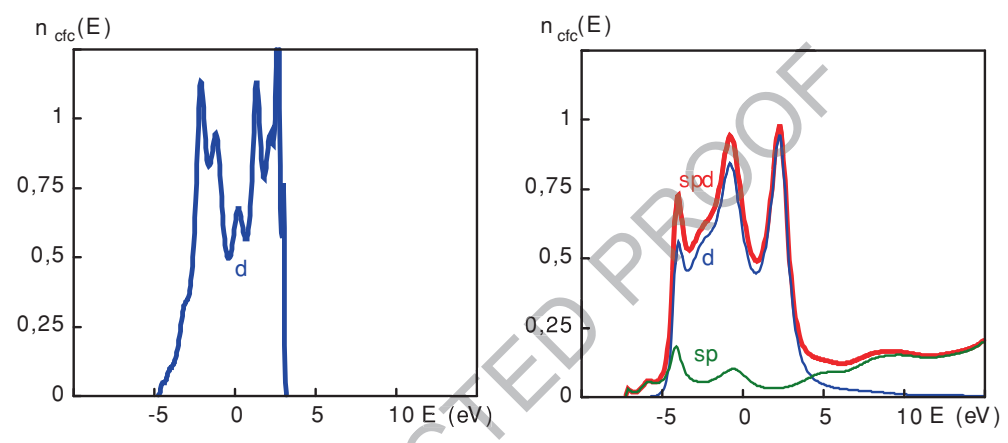

Fig. 6 Influence of $s p$ - $d$ hybridization on the fcc LDOS

bulk material, the coefficients converge towards asymptotic values $a_{\infty}$ and $b_{\infty}$ which are related to band edges, at least for a band without gap [8], so that they can be fitted to band structure calculations.

In this framework, restricting ourselves to $d$-orbitals as commonly admitted for transition metals and using canonical Slater parameters, one obtains archetypal LDOS for the different crystallographic structures shown in Fig. 5. As can be seen, the fcc LDOS is characterized by a high peak in the upper part which is at the origin of the possible occurrence of magnetism (see Sect. 2.5), whereas the bcc one presents a quasi-gap in the middle of the band, which separates bonding states from anti-bonding ones, which tends to favour strongly this structure for half-filled $d$-band elements. However, it is worth noticing that at least at the end of the transition series, it is necessary to take into account the $s$ and $p$ valence electrons and their hybridization with the $d$ ones to get a density of states in good agreement with that derived from DFT calculations [9]. As can be seen in Fig. 6, this strongly modifies the LDOS. 


\subsection{Energetics and Link Between Electronic and Crystallographic Structure}

As usual in any mean-field approximation, the total band energy of the system is not equal to the sum over the one-electron energies $\left(\varepsilon_{\alpha}\right)$, since it counts twice the electron-electron interactions which have to be substracted once. The DFT band energy then writes:

$$
E_{b, 0}^{L D A}=\sum_{\alpha} f_{\alpha} \varepsilon_{\alpha}-\frac{1}{2} \int d \mathbf{r} V^{H}(\mathbf{r}) n(\mathbf{r})+\int d \mathbf{r} n(\mathbf{r})\left(\varepsilon^{x c}[n(\mathbf{r})]-\frac{\partial \varepsilon^{x c}[n(\mathbf{r})]}{\partial n(\mathbf{r})}\right)
$$

The total energy is then obtained by adding the ion-ion contribution to the band one:

$$
E_{0}=E_{b, 0}^{D F T}+\frac{1}{2} \int d \mathbf{r} V^{\text {ion-ion }}(\mathbf{r}) n_{\text {ion }}(\mathbf{r}) \quad \text { with } V^{\text {ion-ion }}(\mathbf{r})=\int d \mathbf{r}^{\prime} \frac{n_{\text {ion }}\left(\mathbf{r}^{\prime}\right)}{\left|\mathbf{r}-\mathbf{r}^{\prime}\right|}
$$

where $n_{\text {ion }}(r)$ is the ionic density: $n_{\text {ion }}(r)=Z \delta(r-n)$, for $Z$ charges at sites $n$.

Within the TB approximation, one can develop this equation, by introducing the local density of states $n_{n}(E)$, and the corresponding charge $N_{n}\left(=\int^{E_{F}} n_{n}(E) d E\right)$ at site $n$ and by assuming charge neutrality $\left(N_{n}=Z_{n}\right.$ ionic charge) [10]. The cohesive energy is then obtained by subtracting the reference of isolated atoms:

$$
E_{c o h}=\sum_{n, \lambda} \int^{E_{F}}\left(E-\varepsilon_{0, \lambda}\right) n_{n}^{\lambda}(E) d E+\frac{1}{2} \sum_{n \neq m} \iint d r d r^{\prime} \frac{Q_{n}(r) Q_{m}\left(r^{\prime}\right)}{\left|r-r^{\prime}\right|}
$$

The first term is the band energy $\left(E_{c o h, b}\right)$ and the second one the pair interaction $\left(E_{c o h, r}\right)$ between neutral atoms with charge density: $Q_{n}(r)=Z_{n} \delta(r-n)-N_{n}(r-n)$. Unfortunately, $E_{c o h, r}$ is not sufficient to account for the repulsive part of the energy. Actually, the TB approximation fails to reproduce part of the repulsion at short distance since it does not account for the non-orthogonality of wave functions on different sites and for the compression of $s p$ electrons which play an important role before the Coulomb repulsion becomes really efficient.

Therefore, in a first step, we will put some emphasis on properties for which the dependence with $d$-band filling suggests that they are mainly driven by the band term.

This is in particular the case of the quasi-parabolic variation of the cohesive energy but also of the atomic volume and bulk modulus experimentally evidenced for each of the transition series (see Fig. 7). This parabolic behaviour is indeed reproduced by calculating the band term of (17) from the previous LDOS. In fact, the integral depending weakly on details of $n(E)$, this band term can be approximated from a schematic rectangular density of states presenting the same second moment (related to the mean width of the LDOS) as the exact one: $E_{c o h, b}=$ $-N_{e}\left(10-N_{e}\right) \beta \sqrt{Z}$ where $\beta$ is an "effective" hopping integral, corresponding to the $Z$ first neighbours. Obviously, some features are not well reproduced in this crude approximation, in particular the asymmetry of the trends which requires a 

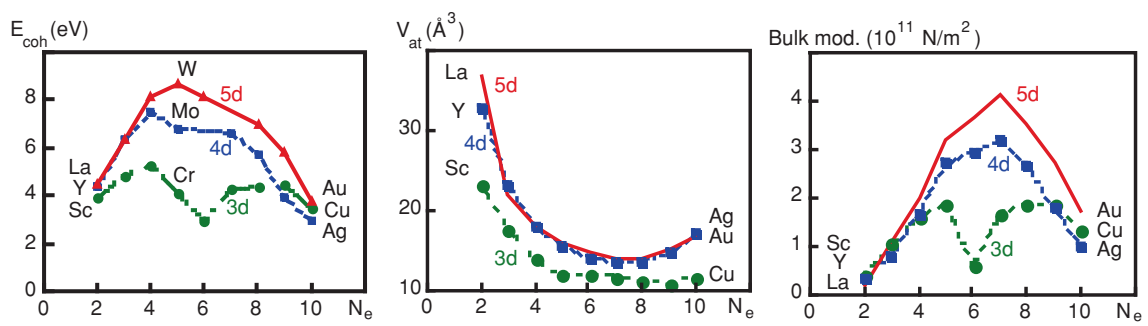

Fig. 7 Experimental variation of the cohesive energy, atomic volume and bulk modulus along transition metal series

Table 1 Crystallographic structure of transition metals

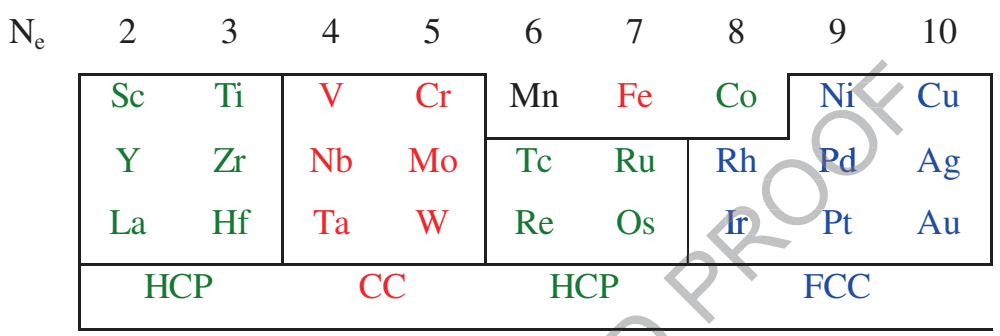

larger number of exact moments and/or including $s p$ - $d$ hybridization to be accounted for. The deep hole of $E_{c o h}$ for the first series is attributed to magnetism.

On the other hand, as can be seen in Table 5.1, the crystalline structure of transition metals is clearly related to the band filling. As shown in Fig. 8 the main trends are correctly reproduced by the TB calculation for the bcc/fcc as well as for hcp/fcc systematics (which of course requires to go beyond second moment since hep and fcc structures are identical up to second neighbours), except for the nearly filled band for which the bcc structure is found instead of fcc. Fortunately, this is corrected if one takes into account $s p$ - $d$ hybridization which, as previously mentioned, plays a major role at the end of transition series. In fact, in the case of the preference for hcp or fcc structure, which involves a weak energy balance and an accuracy of the LDOS beyond second moment, the $s p$ - $d$ hybridization plays a role on the overall trend, consistently with the corresponding influence on the shape of the LDOS.

As shown in Fig. 8, the $s p$-contribution to the energy balance is small as expected since the overall behaviour is driven by that of the partial $d$-band which significantly differs from the non hybridized $d$-band. In the following, it has to be kept in mind that the energetics only depends on the $d$-band, but once distorted by the $s p-d$ hybridization. 
(a)

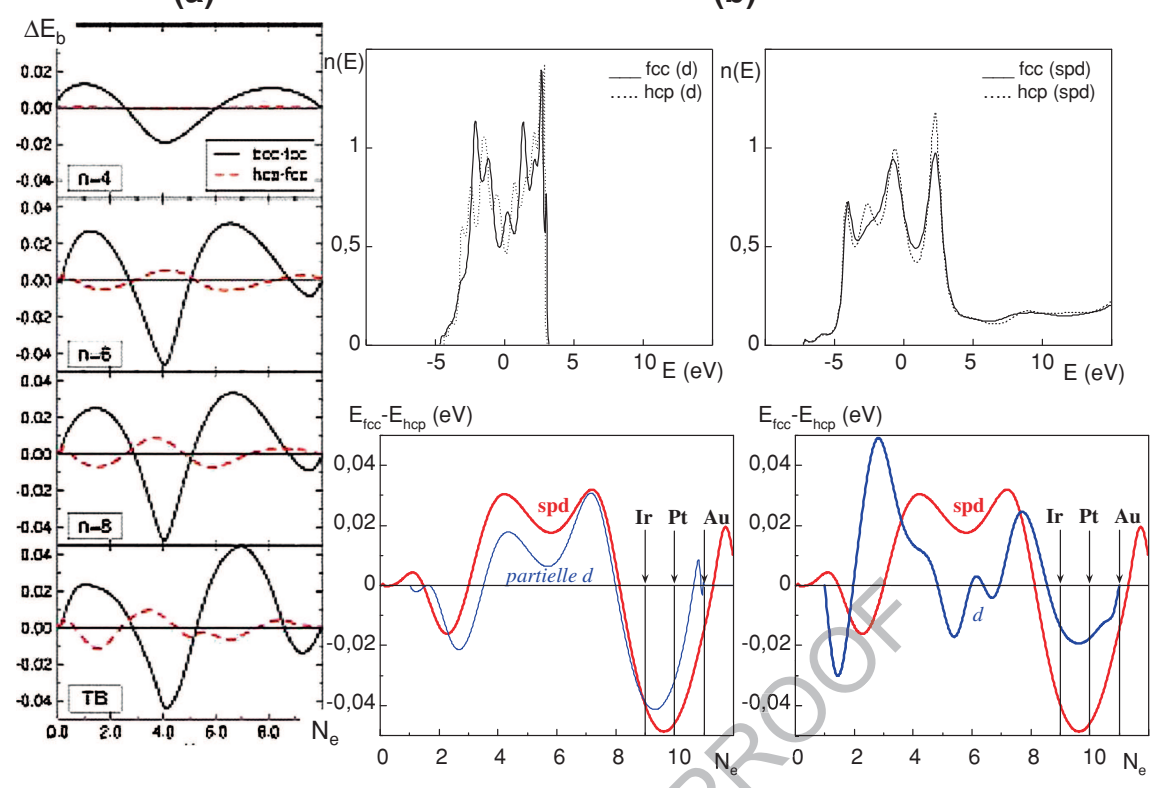

Fig. 8 a Stabilities of fcc relative to bcc $(a)$ and hcp $(b)$ structures in the tight-binding framework (note the two different orders of magnitudes). Effect of $s p-d$ hybridization on the latter competition is also shown in (b). From Refs. [9-11], copyright (2008), with permission from Elsevier

\subsection{Magnetism Within TB Approximation}

For a few metallic elements, the energy of the system can be lowered by shifting the two spin bands, inducing different numbers of electrons with up and down spins $\left(N_{\uparrow}, N_{\downarrow}\right)$, and therefore a finite magnetic moment $\mu=N_{\uparrow}-N_{\downarrow}$. In the framework of collinear magnetism and in absence of spin-orbit coupling, the up and down states are decoupled, so that the sub-systems of up and down electrons can be treated separately, keeping in mind that one must define a single Fermi level $\left(\mathrm{E}_{\mathrm{F}}\right)$ for both spin directions in order to get the right total $d$-band filling $N_{e}=N_{\uparrow}+N_{\downarrow}$. Thus, in a canonical approach, each spin partial LDOS is obtained from the paramagnetic one $n_{O}(E)$ by simply shifting its barycentre $\varepsilon_{0}$ by $\pm \Delta \varepsilon / 2$ :

$$
n_{\uparrow}(E)=n_{0}\left(E+\frac{\Delta \varepsilon}{2}\right) n_{\downarrow}(E)=n_{0}\left(E-\frac{\Delta \varepsilon}{2}\right)
$$

In that case, the magnetic moment is simply given by:

$$
\mu=N_{\uparrow}\left(E_{F}\right)-N_{\downarrow}\left(E_{F}\right)=\int_{E_{F}-\frac{\Delta \varepsilon}{2}}^{E_{F}+\frac{\Delta \varepsilon}{2}} n_{0}(E) d E
$$



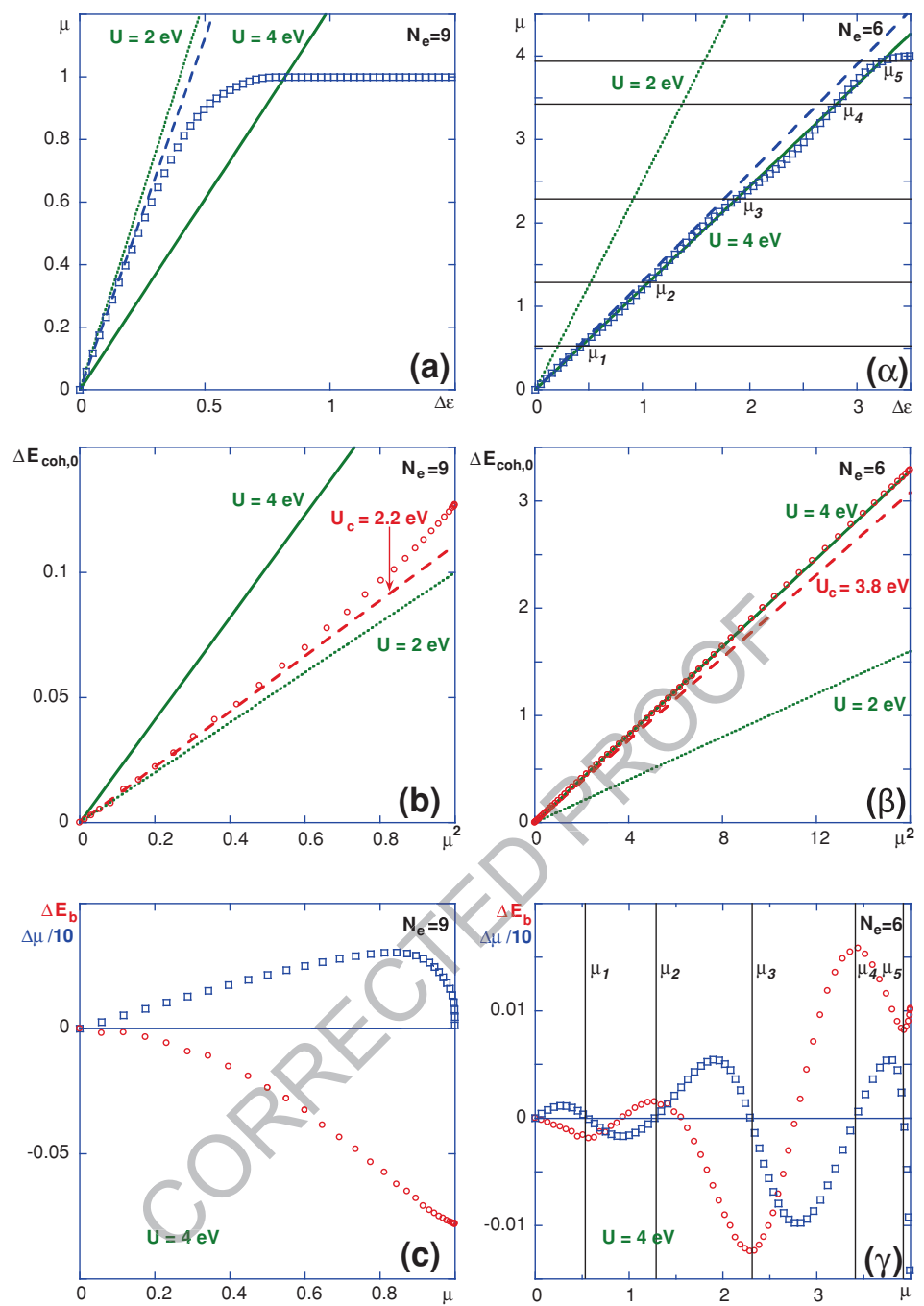

Fig. 9 a, $\alpha$ Self-consistent determination of $\mu$ from the crossing point of Eqs. (18a) (blue dots) and (18c) (green lines) for two values of U. The slope given by Eq. (18b) appears as the dotted blue line $\mathbf{b}, \boldsymbol{\beta} \mu^{2}$-dependence of band term of the energy $\left(\Delta \mathrm{E}_{\mathrm{coh}, 0}\right.$, red dots) given by Eq. (20b) and of the magnetic term $\mathrm{U} \mu^{2} / 20$ for two values of $\mathrm{U}$, green lines. The dotted red line represents the slope given by $1 / 4 \mathrm{n}_{0}\left(\mathrm{E}_{\mathrm{F}}\right)$. (c, $\left.\gamma\right) \mu$-dependence of $\Delta \mu$ Eq. (19) and $\Delta \mathrm{E}_{\mathrm{b}} \mathrm{Eq}$. (20b) for $\mathrm{U}=4 \mathrm{eV}$

which in the limit of weak magnetisation (small $\Delta \varepsilon$ ) reduces to:

$$
\mu \cong n_{0}\left(E_{F}\right) \Delta \varepsilon
$$

This means that the slope at the origin of the $\mu$-curve as a function of $\Delta \varepsilon$ is nothing but the value of the paramagnetic density of states at the Fermi level. 
This is illustrated for an almost filled $\left(N_{e}=9\right)$ in Fig. 9a and for an almost halffilled FCC $d$-band $\left(N_{e}=6\right)$ in Fig. $9 \alpha$.

On the other hand, linearizing the Hamiltonian leads to a self-consistency relation between the $d$-level shifts and the magnetic moment through the Coulomb integral $U$ which writes: $\Delta \varepsilon=U \mu / 5$, giving an other $\Delta \varepsilon$-variation law for $\mu$ :

$$
\mu=\frac{5}{U} \Delta \varepsilon
$$

A self-consistent determination of the magnetic moment then requires finding the crossing points of the two curves as a function of $\Delta \varepsilon$ for the actual value of the $U$ parameter. The asymptotic value of $\mu$ being that which corresponds to a full $u p$ $d$-band filling, the curve given by Eq. (18a) saturates at the value $\mu_{s}=10-N_{e}$, being then below the line given by Eq. (18b). That means that it can cross the curve given by Eq. (18c) only if the so-called Stoner factor $S_{0}=U n_{0}\left(E_{F}\right) / 5$ is larger than unity (Stoner criterion). As can be seen, for a value of $U$ which satisfies this criterion (here $U=4 \mathrm{eV}$ ) this crossing point only exists for the saturation value $\left(\mu_{s}=1\right)$ for $N_{e}=9$, which corresponds to the limit of strong ferromagnetism (full up band). At the opposite, different crossing points $\left(\mu_{1}, \ldots, \mu_{5}\right)$ are found below the saturation value $\left(\mu_{s}=4\right)$ for $N_{e}=6$ (weak ferromagnetism), in agreement with experiments. This is still clearer in Fig. $9(\mathrm{c}, \gamma)$, in which is plotted (for $U=4 \mathrm{eV}$ ) the difference:

$$
\Delta \mu=\int_{E_{F}-\frac{\Delta \varepsilon}{2}}^{E_{F}+\frac{\Delta \varepsilon}{2}} n_{0}(E) d E-\frac{5}{U} \Delta \varepsilon
$$

In that case, the equilibrium value of $\mu$ is the one that which minimizes the band energy $E_{b}$ :

$$
E_{b}(\mu)=\int^{E_{F}} E n_{\uparrow}(E) d E+\int^{E_{F}} E n_{\downarrow}(E) d E-N_{e} \varepsilon_{0}+\frac{5}{4} U\left(\frac{\mu}{5}\right)^{2}
$$

where the last two terms account for the double counting of interactions in the oneelectron term. Taking advantage of the self-consistent relation (18c), this energy also writes:

$$
E_{b}(\mu)=E_{c o h, 0}\left(N_{\uparrow}\right)+E_{c o h, 0}\left(N_{\downarrow}\right)-\frac{1}{20} U \mu^{2}
$$

with: $E_{c o h, 0}\left(N_{\sigma}\right)=\int^{E_{F}^{\sigma}} E n_{0}(E) d E-N_{\sigma} \varepsilon_{0} ; E_{F}^{\sigma}=E_{F} \pm \frac{\Delta \varepsilon}{2}$ depending on $\sigma=\uparrow, \downarrow$.

The gain (or loss) in energy due to magnetism for a given d-band filling is then given by:

$$
\Delta E_{b}(\mu)=\Delta E_{c o h, 0}\left(N_{e}, \mu\right)-\frac{1}{20} U \mu^{2}
$$




$$
\Delta E_{c o h, 0}\left(N_{e}, \mu\right)=E_{c o h, 0}\left(N_{\uparrow}\right)+E_{c o h, 0}\left(N_{\downarrow}\right)-2 E_{c o h, 0}\left(N_{e}\right)
$$

As can be seen, for small values of the magnetic moment, the first term, independent on $U$, is nothing but the second derivative of the curve $E_{c o h, O}\left(N_{e}\right)$ at the considered band filling. From the convexity of this curve, it appears that this band term is positive and then disfavours magnetism whereas the second (magnetic) term is explicitly negative and then favours its occurrence. In the limit of small values of the magnetic moment $\mu$ or in the particular case where the LDOS does not vary much around the Fermi level, the equations (20b) can be developed into:

$$
\Delta E_{b}(\mu) \cong \frac{\mu^{2}}{20}\left(\frac{5}{n_{0}\left(E_{F}\right)}-U\right)
$$

The balance between these two terms then gives the sign of the slope of $\Delta E_{b}(\mu)$ curve at the origin $(\mu=0)$. This allows to recover the previous Stoner criterion, the critical value of $U$ being $U_{c}=5 / n_{O}\left(E_{F}\right)\left(2.2 \mathrm{eV}\right.$ for $N_{e}=9$ and $3.8 \mathrm{eV}$ for $\left.N_{e}=6\right)$. However, finding the actual value of the magnetic moment requires to go beyond such approximations (small $\mu$ or LDOS almost constant around the Fermi level). One sees in Fig. $9(\mathrm{~b}, \beta)$ that the band term $\Delta E_{c o h, O}$ indeed deviates from a simple $\mu^{2}$ behaviour. This is still more apparent in Fig. $9(\mathrm{c}, \gamma)$ in which we plot $\Delta E_{b}$ given by Eq. (20b). As expected from the self-consistent treatment of $\mu$ (Fig. 9(a, $\alpha), \Delta E_{b}$ is decreasing up to the saturation value $\mu_{s}$ for $N_{e}=9$ (strong ferromagnetism), whereas one recovers five extrema (with three minima), corresponding to the five crossing points of Fig. $9 \alpha$ for $N_{e}=6$ (weak magnetism). In the latter case, the equilibrium value corresponds to the absolute minimum $\left(\mu_{3}=2.3\right)$. The relative stabilities of the three minima is obviously strongly dependent on the value of $U$ (in the range between $U_{c}$ and $4.2 \mathrm{eV}$ ), or equivalently from variations of the LDOS with respect to its equilibrium shape, due to variation of interatomic distances (e.g. epitaxial growth, defects, dilation), which implies that the magnetisation could change under small variations of experimental conditions.

\section{Pure Metal Surfaces and Clusters}

\subsection{Surface LDOS, Charge Self-Consistency and Atomic Level Shifts}

If one neglects the crystal field $\alpha_{\lambda}$, two bulk parameters should vary at the surface: first the hopping integrals $\beta_{n m}^{\lambda \mu}$ that we will assume unchanged at the surface (no relaxation), then the effective $\lambda$ levels $\varepsilon_{\lambda}$. Let us first assume that the latter is also unchanged at the surface. In that case, the first effect of bond breaking $(\Delta Z)$ is to narrow the LDOS at the surface, due to simple second moment arguments. 

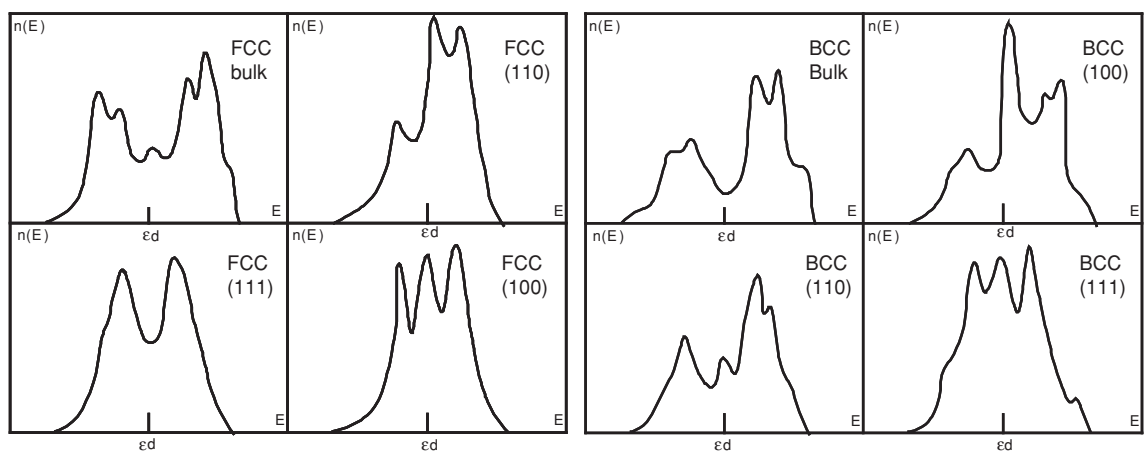

Fig. 10 Surface LDOS for fcc (left) and bcc (right) low index surfaces

(a)

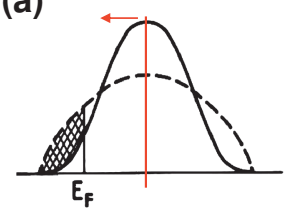

(b)
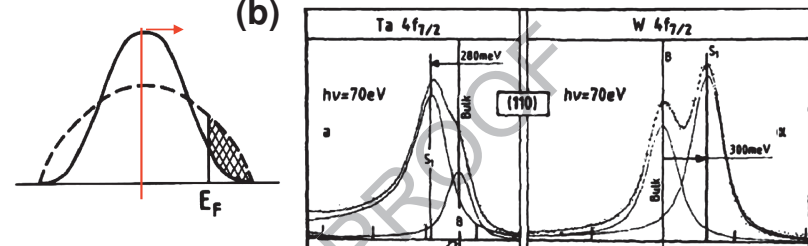
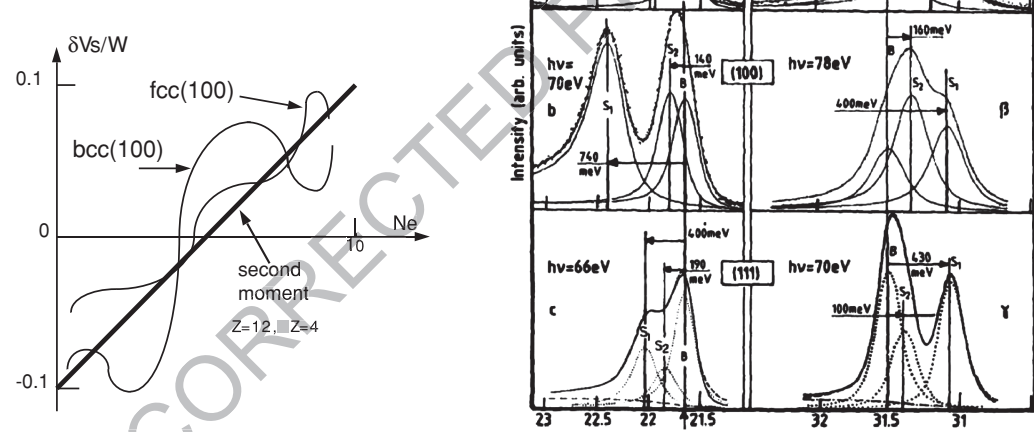

Fig. 11 a Variation with the $d$ band filling (for realistic and rectangular densities of states) of the surface valence level shifts induced by the local charge neutrality requirement (from Ref. [12]). b Experimental surface core level shifts for Ta and W low index surfaces (from Ref. [13], copyright (1985), with permission from Elsevier)

In addition, as can be seen in Fig. 10, the LDOS corresponding to the most open surfaces (namely (110) and (100) for fcc, (100) and (111) for bcc) present quasi surface states, which vanish beyond the surface layer.

However, once the Fermi level is fixed by the bulk density of states, keeping $\varepsilon_{\lambda}$ unchanged at the surface (Fig. 11a) leads to an unrealistic electronic charge transfer which must obey some self consistent rule, since this charge redistribution modifies the potential and then shifts $\varepsilon_{\lambda}$ by $\delta \varepsilon_{\lambda, s}$.

This is illustrated in Fig. 11 for a single $d$-band. As can be seen the dependence of $\delta \varepsilon_{d, s}$ with $d$-band filling follows some general trends: it changes sign near the 

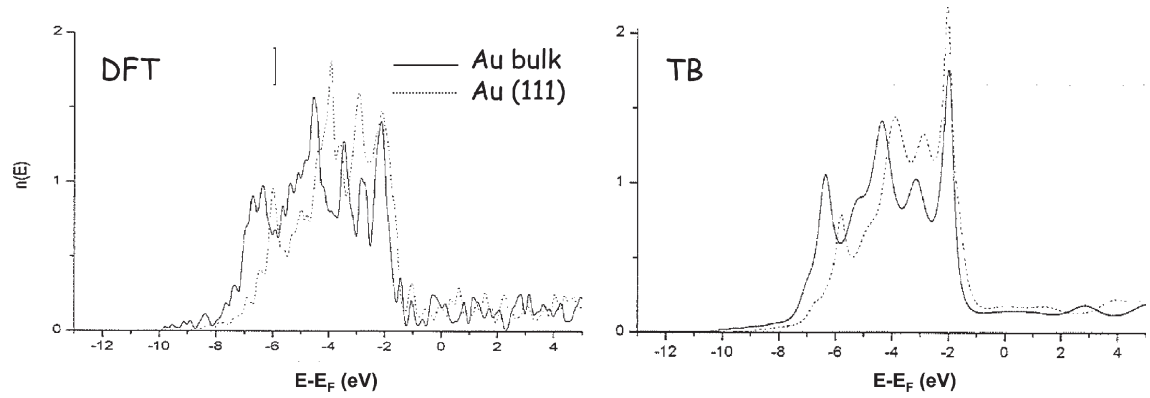

Fig. 12 Au density of states, calculated either by DFT (SIESTA) (left) or in the tight-binding framework (right) with $s p$ - $d$ hybridization with 25 exact couples of coefficients. From Ref. [15]

middle of the series (between $\mathrm{W}$ and $\mathrm{Ta}$ in the $5 \mathrm{~d}$ series), its absolute value increases with the number of broken bonds (fcc: $\left|\delta \varepsilon_{d, 110}\right|>\left|\delta \varepsilon_{d, 100}\right|>\left|\delta \varepsilon_{d, 111}\right|$ ), with a maximum value of about $\mathrm{W} / 10$ (W: $d$-band width). Finally, it is worth mentioning that this $d$ level shift is almost rigidly followed by the core levels, which is confirmed experimentally by core level spectroscopy [13].

Let us note that this charge neutrality condition at the surface is confirmed by ab initio calculations. More precisely the DFT calculations even show that, when considering $s p-d$ hybridization, charge neutrality has to be achieved, not only for each inequivalent site, but also for each orbital [14]. As can be seen in Fig. 12 for the $\mathrm{Au}(111)$ surface the resulting LDOS's compare satisfactorily to those of DFT calculations. Moreover, $s p-d$ hybridization leads to surface energies in better agreement with experiments [15].

\subsection{Relaxations and Reconstructions: Second Moment Approximation}

Due to the broken bonds, the surface atoms can undergo displacements with respect to their bulk positions. In all cases, there is at least a vertical relaxation, which is experimentally known to be inwards (contraction of the first interlayer distance) for transition metals. In order to model this behaviour, we need not only the band part of the energy but also the repulsive one. Unfortunately as already mentioned, TB does not give such a repulsive part. To go beyond this difficulty, the idea is to build a semi-phenomenological TB model in which the band part, coming from the electronic structure, has a many-body character whereas the repulsive one is a pairwise potential fitted to some physical properties. Subtracting as usual (Sect. 2.4) the contribution due to this correction which is counted twice, the Eq. (17) reduces to: 


$$
E_{c o h}=\sum_{n, \lambda} \int^{E_{F}}\left(E-\varepsilon_{0, \lambda}\right) n_{n}^{\lambda}\left(E, \delta \varepsilon_{\lambda}\right) d E-\sum_{\lambda=s, p, d} N_{0}^{\lambda} \delta \varepsilon_{\lambda}+A \sum_{R} e^{-p\left(\frac{R}{R_{0}}-1\right)}
$$

Since the integral does not depend on details of $n(E)$, the band term can be calculated from a schematic rectangular density of states under the single assumption that it has the same second moment (SMA) as the exact one, which leads to $[6,16]$ :

$$
E_{c o h}=-\beta \sqrt{\sum_{R} e^{-2 q\left(\frac{R}{R_{0}}-1\right)}}+A \sum_{R} e^{-p\left(\frac{R}{R_{0}}-1\right)}
$$

where $\beta$ is an "effective" hopping integral, corresponding to the $1^{\text {st }}$ neighbour distance $R_{O}$. In practice, for a given metal, the four parameters $(A, \beta, p, q)$ are determined by fitting experimental quantities such as the cohesive energy, lattice parameter and elastic constants or the so-called universal equation [17]. Note that this SMA-potential is similar to those derived elsewhere within the Embedded Atom Model (EAM [18]) or within the Glue Model [19]. Its main advantage compared to the latter is its physical transparency which clearly shows its limitations... and then its possible improvements (increasing the number of exact moments).

The first success of SMA potentials is to reproduce the inwards surface relaxation, which is found to be of the order of a few percents and proportional to the number of broken bonds, contrary to simple pair potential models which predict an outwards relaxation [13]. This comes from the stronger decrease with coordination $Z$ of the repulsive term compared to the attractive one $(\sim \sqrt{ } Z)$. One has then to use such many body potentials to study surface atomic rearrangements.

In some cases, not only vertical but also lateral atomic rearrangements can occur, changing the two-dimensional periodicity and leading to so-called surface reconstructions. In those cases, one observes trends which can be either along the transition series (zig-zag reconstruction of the (100) face of bcc crystals occurring for column VIa but not for $\mathrm{V}_{\mathrm{a}}$ ) or along a column ((110) missing row reconstruction or pseudohexagonal densification of (100) fcc surfaces which only occurs in the $5 d$ series). Both trends are well interpreted in the framework of TB calculations. The physical origin of the column sequence for fcc reconstructions, attributed to relativistic effects, can be taken into account within SMA potentials through the increase of the $q$ parameter from the 1 st series to the 3rd one [20]. On the other hand, understanding the zig-zag reconstruction requires going beyond second moment arguments. Indeed, as illustrated in Fig. 13a, it is due to the broadening of the quasi-atomic surface peak of the local bcc (100) density of states under the lattice distortion, which leads to an energy gain for $d$ band filling around 5 (middle of the series) [21]. A similar detailed description of the LDOS is also required to study the possible occurrence of an hcp/ fcc staking fault at the surface of late transition elements, in which case one also needs to account for $s p$ - $d$ hybridization [9]. The corresponding fault energy is found to be in good agreement with DFT calculations (see Fig. 13b). Its weak value for Au is consistent with the experimental observation of the herringbone reconstruction which leads atoms of the (111) surface layer to occupy both the hep and the fcc sites. 
(a)

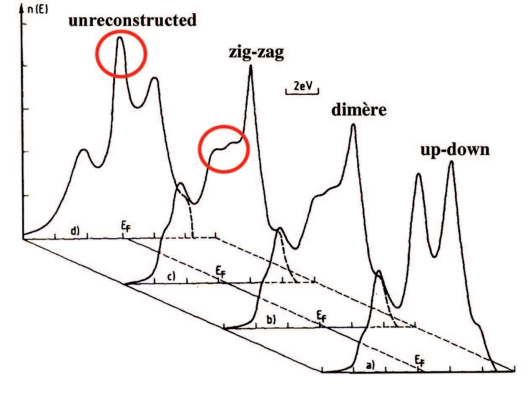

(b)

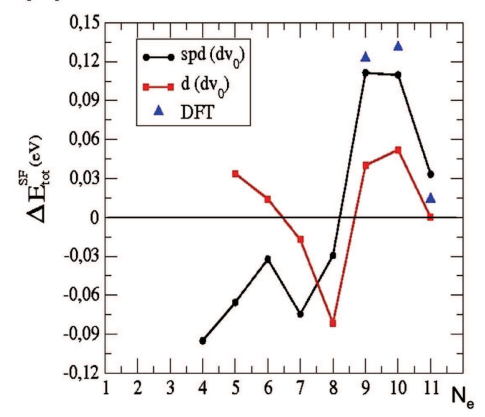

Fig. 13 a Influence of the dimer reconstruction on the (100) LDOS of bcc metals (from Ref. [21]). b Variation of the surface fcc-hcp stacking fault energy with $\mathrm{d}$ band-filling from self-consistent TB and DFT calculations (from Ref. [9], copyright (2008), with permission from Elsevier)

\subsection{Monometallic Clusters: LDOS}

Obviously, ab initio methods are particularly suited to the study of clusters with very small sizes but become very cumbersome when these sizes reach those which are useful for catalysis purposes (more than 100 atoms). The TB method is then very useful, since it describes the electronic structure in a wide range of sizes, and is able to give reliable site energies. Technically, for a finite cluster, the coefficient $b_{n} \rightarrow 0$ beyond a given level so that the continued fraction see Eq. (13) is truncated leading to a discrete spectrum for the LDOS of any site. This is illustrated for the central site of a 55 atoms cuboctahedron in Fig. 14.

Here also, when interested in elements of the end of transition series, it is necessary to take into account the $s p$ - $d$ hybridization and to perform a self-consistent treatment of the relation between charge and potential. This is achieved as for surfaces by shifting the $\lambda$-levels in a different way on each inequivalent site, following the same neutrality rule per site and per orbital [23]. The variation of these shifts for the different orbitals and sites as a function of their coordination numbers is also plotted in Fig. 15a. Note that theses shifts can be directly related to the activity of the corresponding sites [24], reflecting the high activity of the low coordination sites.

The resulting surface LDOS present a band width which decreases with the site coordination (from facets to edges and vertices) and are significantly modified near the Fermi level depending on the site. Note that, in view of the arguments developed in Sect. 2.5, this band narrowing could induce occurrence of magnetism for clusters of elements which are non magnetic in the bulk. Moreover the cluster symmetry has a strong influence on the density of states. The influence of size and structure is illustrated in Fig. 15b where we plot the total density of states for cuboctahedral and icosahedral (Ih) clusters, by taking the average of the densities 


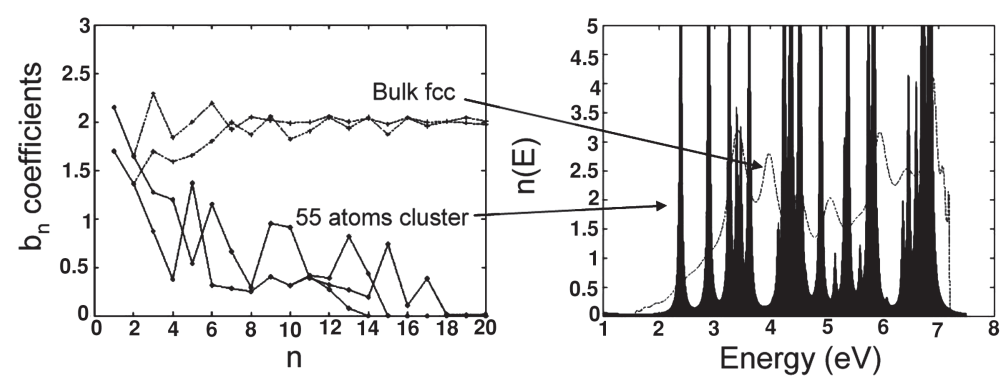

Fig. 14 Variation of the $b_{n}$ coefficients for a bulk fcc structure and the central site of a 55-atoms cuboctahedron (left) and corresponding LDOS (right). From Ref. [22]

(a)

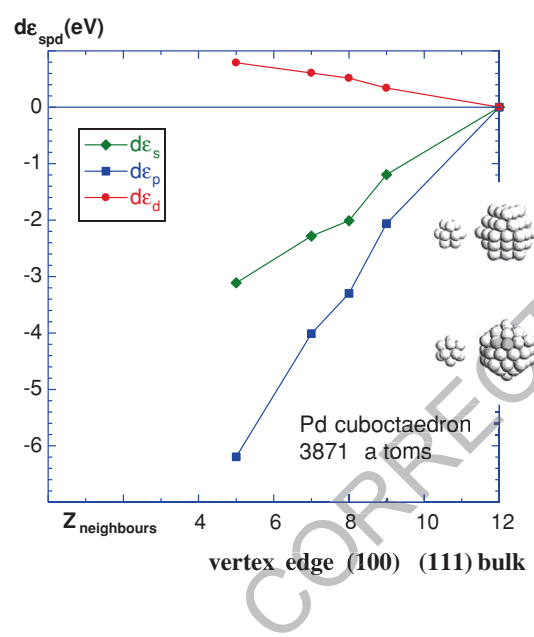

(b)

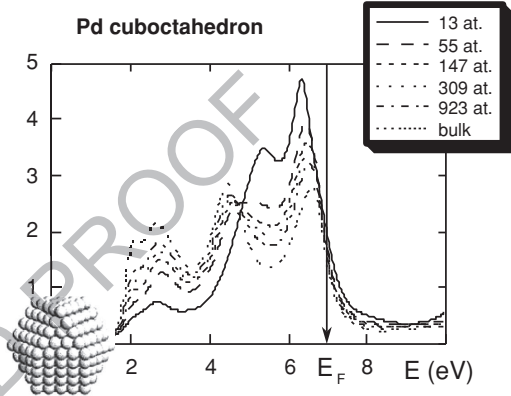

(c)

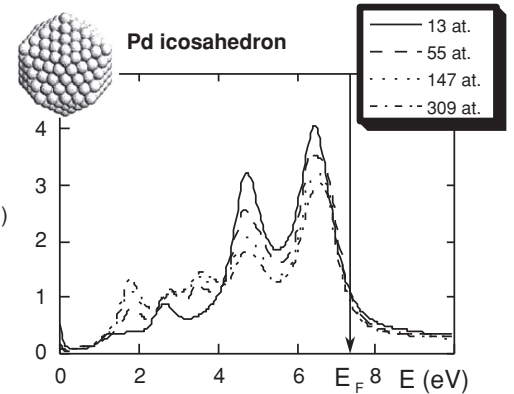

Fig. 15 a Core level shifts on the various surface sites of a 3,871 Pd cuboctahedron. b Variation with size of the average LDOS for Pd cuboctahedra and icosahedra from self-consistent TB calculations. From Ref. [23], copyright (1996), with permission from Elsevier

on inequivalent sites (vertices, edges, facets and core) weighted by the corresponding number of sites [23].

From energetic calculations performed by Quenched Molecular Dynamics within the SMA model, it appears that the competition between fcc and Ih structures is strongly element dependent. Thus, while Ih Ag clusters are found to be stable until 309 atoms, Au clusters recover their bulk fcc morphology (Wulff polyhedron) beyond 13 atoms. This critical size strongly depends on the local relaxation which differs remarkably between fcc-type and Ih clusters. The 
latter adopt a very inhomogeneous atomic relaxation profile, in which the contraction of the intershell distance is not limited to the surface shell (as in fcc-type structures) but is also present for the inner shells. More precisely, a considerable core contraction is found in the Ih case, which increases as a function of the cluster size [25]. A spectacular consequence of this contraction of the inner shells for the Ih structure is the existence of a strong compressive pressure in the core which can be relaxed by introducing constitutive vacancies [25]. The stability of these vacancies increasing with the cluster size, a fourfold tetrahedral shaped cavity becomes even more stable than the single one, but beyond the morphological transition to fcc-type structures. Taking into account the stability domain of the Ih relatively to fcc structure, there should then exist a stability range of size for $\mathrm{Cu}$ and Ag icosahedra with a central constitutional vacancy but not for Au ones. Let us recall however that SMA potentials are less suited than DFT calculations to model too small clusters. Thus, for 13 atoms, DFT calculations find structures which are neither Ih nor fcc [26].

\section{Bulk Alloys $\mathbf{A}_{\mathbf{c}} \mathbf{B}_{1-\mathrm{c}}$ : Link Between Electronic and Chemical Structure}

\subsection{Influence of Chemical Ordering on LDOS}

Extending the Tight-Binding Hamiltonian to the case of a binary alloy $\mathrm{A}_{\mathrm{c}} \mathrm{B}_{1-\mathrm{c}}$ requires to make its parameters depend on chemical configuration $\left\{p_{n}^{i}\right\}, p_{n}^{i}=1$ if site $n$ is occupied by atom of $i$-species $(i=\mathrm{A}, \mathrm{B})$ and $p_{n}^{i}=0$ if not, through the relation:

$$
\begin{gathered}
H=H_{d}+H_{n d} \\
H_{d}=\sum_{n, \lambda}|n, \lambda\rangle \varepsilon_{n \lambda}\langle n, \lambda| \varepsilon_{n}=\sum_{i=A, B} p_{n}^{i} \varepsilon_{n \lambda}^{i} \\
H_{n d}=\sum_{n, m, \lambda, \mu}|n, \lambda\rangle \beta_{n m \lambda \mu}\langle m, \mu| \beta_{n m \lambda \mu}=\sum_{i, j=A, B} p_{n}^{i} p_{m}^{j} \beta_{n m \lambda \mu}^{i j}
\end{gathered}
$$

The parameter $\varepsilon_{\lambda}^{i}$ which appears in the diagonal contribution $H_{d}$ is the barycentre of the partial $i \lambda$-LDOS, projected on the $\lambda$-orbital of atom of type $i$, while the off-diagonal contribution $H_{n d}$ involves the hopping integral between the $\lambda$-orbital at site $n$ occupied by an atom of type $i$ and $\mu$-orbital at site $m$ occupied by an atom of type $j$. Thus the modifications undergone when two elements $(\mathrm{A}, \mathrm{B})$ are mixed into an $A_{c} B_{1-c}$ alloy comes from two effects [10]. The first one, which is called diagonal disorder effect, is induced by the difference in energy between the barycentres of the valence (essentially $d$ ) bands of the A and B pure elements, i.e., the corresponding atomic levels $\varepsilon_{d, 0}^{A}$ and $\varepsilon_{d, 0}^{B}$ (the index 0 refers to the pure bulk value), and it is quantified by the parameter $\delta_{d, 0}=\varepsilon_{d, 0}^{A}-\varepsilon_{d, 0}^{B}$. The second 
(a)

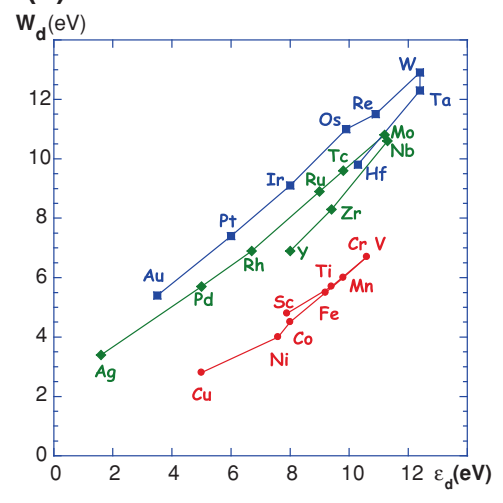

(b)

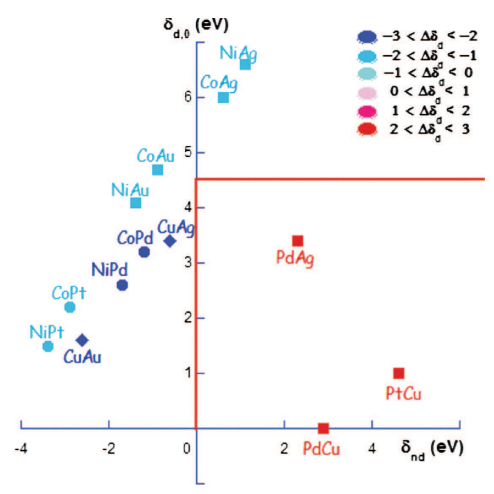

Fig. 16 a Correlated variations of the $d$ atomic level $\varepsilon_{d, 0}^{i}$ and effective bandwidth $W^{i}(\sim-8 d d \sigma)$ interpolated from ab initio band structure calculations (results from Ref. [27]) along the three transition metal series from which are derived $\left(\delta_{d, 0}, \delta_{n d}\right)$ for each alloy. (b) $2 \mathrm{D}\left(\delta_{d, 0}, \delta_{n d}\right)$ map used to classify the variation $\Delta \delta_{d}$ of $\delta_{d, 0}$ induced by the charge neutrality condition (from Ref. [32], copyright (2011) by The American Physical Society)

one, which is called off-diagonal disorder effect, comes from the difference between the hopping integrals for pure $\mathrm{A}$ and $\mathrm{B}$ elements, and therefore between the pure $\mathrm{A}$ and $\mathrm{B}$ valence bandwidths $W^{A}$ and $W^{B}$ which are directly related (see Sect. 2.3), so that it is quantified by the parameter $\delta_{n d}=W^{A}-W^{B}$. The relative magnitude of these two parameters actually drives the redistribution of the electronic states with respect to those of pure elements and therefore induces the new properties of the alloy with respect to those of the two isolated elements.

The values of $\varepsilon_{d, 0}^{i}$ and $W^{i}$ have been interpolated from ab initio calculations of band structure and tabulated for all transition elements by D. A. Papaconstantopoulos [27]. Their systematic variation along the three transition metal series, i.e., as a function of the $d$ band filling $N_{d}^{i}$, is displayed in Fig. 16. As can be seen from these trends, none of the diagonal and off-diagonal variations seems to prevail, even though it has often been argued that the former prevailed on the latter [10]. In addition both variations are clearly correlated, which means that one can expect both effects to be important or not in the same time for a given system.

Once a given alloy has been characterized by a set of parameters $\left(\delta_{d, 0}, \delta_{n d}\right)$, let us detail how to calculate more precisely the density of states for an alloy. In the ordered case, the same methods (continued fraction, recursion) can be used as for pure elements, taking just into account the ordered configuration of $\mathrm{A}$ and $\mathrm{B}$ atoms to assign the levels $\varepsilon_{\lambda}^{i}$ and the hopping parameters $\beta_{n m \lambda \mu}^{i j}$. The situation is more complicated for a disordered system since it requires to calculate the average value, over all configurations, of $n(E)$ and therefore of $G(E)$. In the absence of offdiagonal disorder $\left(\delta_{n d}=0\right)$, i.e., assuming that $\beta_{n m \lambda \mu}^{i j}=\beta_{n m \lambda \mu}$, the off-diagonal part of the Hamiltonian $\left(H_{n d}\right)$ is the same as for the pure elements, and only the 
diagonal part $\left(H_{d}\right)$ depends on the chemical configuration through $\varepsilon_{n}$. If one notes $G_{0}=\left(z I-H_{n d}\right)-1$ the Green function for the pure metal, one can calculate the average Green function $G$, within a mean-field approximation, by introducing an effective local potential $\Sigma(z)$ such as:

$$
G(z)=\left(z-H_{0}-\Sigma(z)\right)^{-1} \text { with } \Sigma(z) \approx \sum_{n}|n\rangle \sigma(z)\langle n|
$$

which means that in the average medium, the levels $\varepsilon_{\lambda}^{i}$ are replaced by $\sigma(z)$ at each site. This effective potential can be determined by a self-consistency condition which imposes that fixing the occupancy of a site and then making the average on this site would lead to recover the same potential. This is the Coherent Potential Approximation (CPA) [28], which leads to the condition:

$$
\sum_{i=A, B i} c^{i} t^{i}=0 \text { with } t^{i}=\frac{\varepsilon^{i}-\sigma}{1-\langle n|G(z)| n\rangle\left(\varepsilon^{i}-\sigma\right)}
$$

which is self-consistent since the Green function in the disordered state $\langle n|G(z)| n\rangle$ depends on $\sigma(z)$ through the relation: $\langle n|G(z)| n\rangle=G_{0}(z-\sigma(z))$ which only requires the knowledge of the Green function of the pure element. The alloy densities of states obtained in this way (recursion method for ordered system, CPA and continued fraction for disordered ones) [29] are in good agreement with those obtained by LMTO calculations [30].

If one uses canonical parameters for the pure elements, the electronic structure of the alloy depends on $c$ and $\delta_{d, 0}$. In the case of weak diagonal disorder, perturbations at the lowest order lead to a density of states for the disordered alloy which is almost the same as that of the pure metal, but centred on the average level $\bar{\varepsilon}=c \varepsilon^{\mathrm{A}}+(1-c) \varepsilon^{B}$ with an average bandwidth $\bar{W}=c W^{\mathrm{A}}+(1-c) W^{B}$. On the contrary, for a strong diagonal disorder, a gap is opened since electronic states for the alloy have to lie between the bounds for the pure metals [10]. One can then analyze schematically the effect of chemical ordering in the following way. In the case of phase separation, the alloy density is the average of those of the pure metals whereas the sub-bands are narrower in the case of perfect order since the number of neighbours of the same type is reduced. For disordered systems, the width is in between but tails are present due to the finite probability of finding pure A and B clusters of any size. Finally, the total LDOS can be decomposed into its partial contributions projected on each element. All these qualitative behaviours are illustrated in Fig. 17.

\subsection{Charge Self-Consistency and Atomic Level Shifts}

It is worth pointing out that, up to now, a specific alloy has only be defined by the set of values of $\left(\delta_{d, 0}, \delta_{n d}\right)$ and implicitly its crystallographic structure, without 


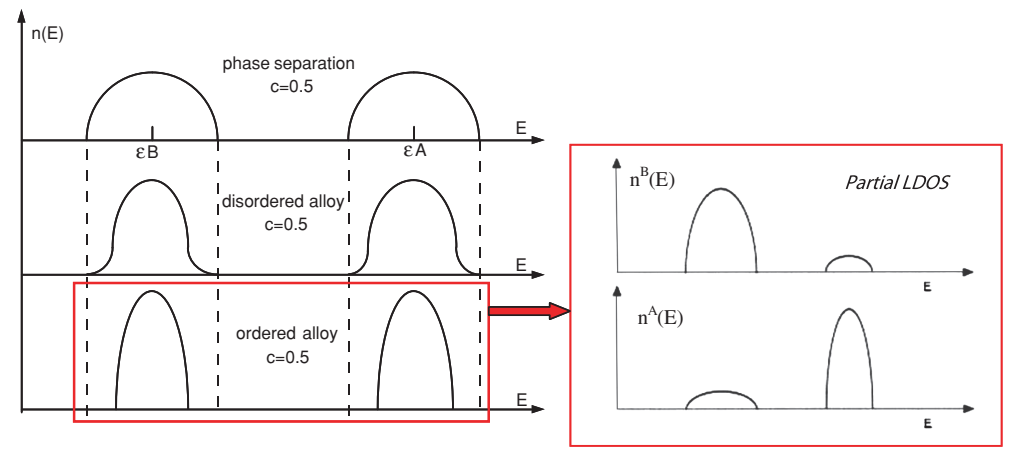

Fig. 17 Schematic variation of the density of states upon alloying for strong diagonal disorder. From Ref. [10]

resorting to the respective number of electrons of each species per orbital $N_{\lambda}^{i}$. However starting from the values in the pure elements $N_{\lambda, 0}^{i}$ (which are consistent with the respective atomic levels $\varepsilon_{\lambda, 0}^{i}$ ) while fixing a common Fermi level in the alloy leads to some charge transfer between species and orbitals. This in turn modifies the barycentres of the partial sub-bands $\varepsilon_{\gamma}^{i}$, the procedure having to be iterated up to convergence towards their self-consistent values. Thus, the parameter which actually drives the variation of the electronic structure between the alloy and its pure constituents is not $\delta_{d, 0}$ but instead the difference between the barycentres of the two alloy partial sub-bands with respectively A and B characters: $\delta_{d}=\varepsilon_{d}^{A}-\varepsilon_{d}^{B}$.

From the experimental point of view, as previously stated for surfaces, it is difficult to identify such a shift of the $d$-bands under alloying due to their dispersion, but it is easier to follow the corresponding shift of the core levels. However, although a lot of work has been made in the case of surface core-level shifts in pure metals [13], only a few have been achieved for alloys. In the latter case, the most documented work in the literature is due to Olovsson et al. [31] who calculated core level binding energy shifts for various disordered alloys within density functional theory (DFT) using the coherent potential approximation (CPA). This allowed them to get good agreement with experimental data, but not a unified physical picture permitting to predict the general behaviour of any alloy.

The main difficulty is then to determine the effective atomic level $\varepsilon_{\lambda}^{i}$ for each partial $i$-sub-band and $\lambda$-orbital in order to ensure the charge self-consistency. This requires shifting these levels for each orbital $\lambda$ with respect to those in the bulk by a value $\delta \varepsilon_{\lambda}^{i}$ in order to satisfy a given rule on the different band fillings per orbital and species $N_{\lambda}^{i}$. The local charge neutrality rule per site and per orbital already justified for surfaces with only $d$ electrons [14] detailed in Sect. 3.1 has been extended to a neutrality per chemical species in the case of bimetallic compounds [15]. Applying such a rule implies to ensure partial charge neutrality of each $(s, p, d)$ orbital and to find for each one the appropriate band shift $\delta \varepsilon_{\lambda, 0}^{i}$ for each 

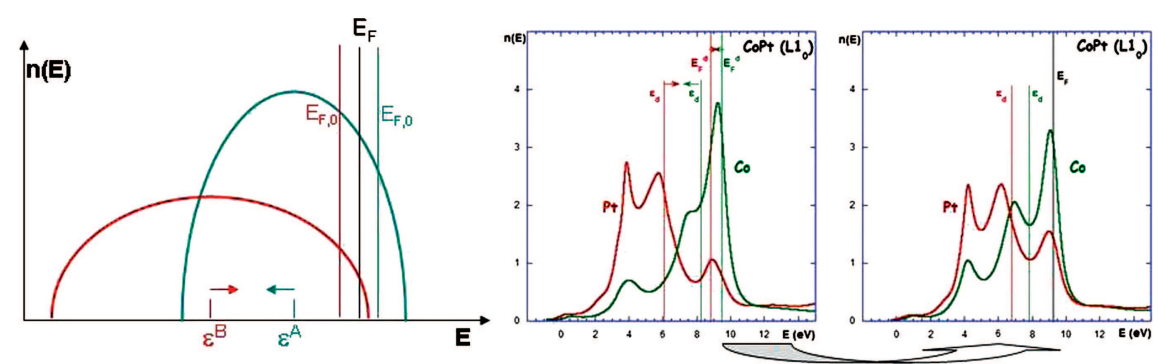

self-consistency
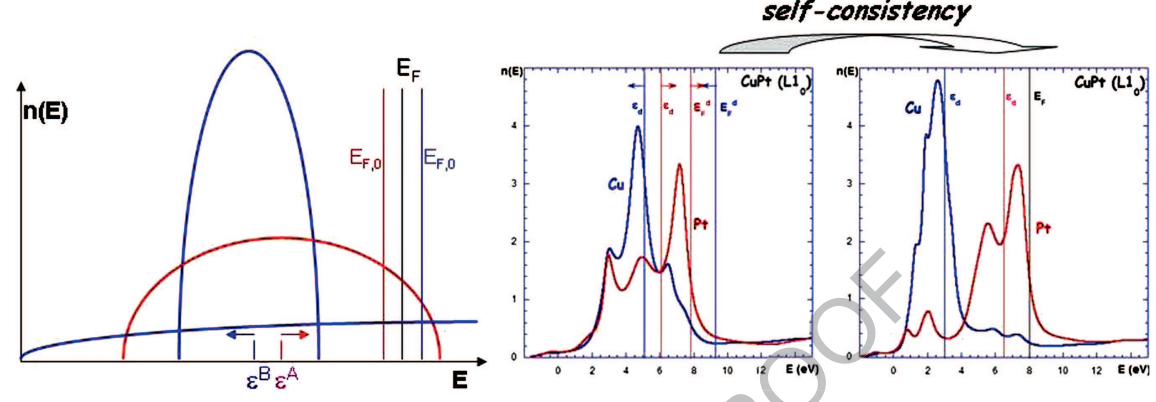

Fig. 18 Schematic (left) and TB (right) LDOS's in (up) CoPt and (down) PtCu systems in the $L 1_{O}$ phase. The TB LDOS are presented before and after the charge self-consistency treatment

element. As an example, this procedure has been applied to predict the behaviour of late transition series alloys constituted of one element of the first transition series $(\mathrm{Co}, \mathrm{Ni}, \mathrm{Cu})$ and the other elements of the second $(\mathrm{Pd}, \mathrm{Ag})$ and third $(\mathrm{Pt}, \mathrm{Au})$ series, in terms of the single diagonal and off-diagonal disorder parameters, which have been the subject of X-ray spectroscopy experiments and in addition are extensively studied for their peculiar properties in various applications, and in particular as nanoalloys. Adopting as a general rule that we denote respectively A and B two elements such as $\delta_{d, 0}=\varepsilon_{d, 0}^{A}-\varepsilon_{d, 0}^{B}>0$, the corresponding values of the variations $\Delta \delta_{d}=\delta_{d}-\delta_{d, o}$ under self-consistency are displayed in the $2 \mathrm{D}\left(\delta_{d, 0}, \delta_{n d}\right)$ mapping of Fig. 16 [32].

As can be seen, almost all the systems present a decrease of the diagonal disorder parameter between 30 and 6\%. Only two systems present an increase of this parameter, which fall in a well delimited region of this map $\left(\delta_{d, 0} \cdot \delta_{n d}>0\right.$, $\left.\delta_{d, 0}<\delta_{d, c}\right)$. The widely commonly encountered decrease of the diagonal parameter under self-consistency is indeed expected [33] and can be intuitively understood by considering that, in the absence of self-consistent treatment, the only way to ensure $d$ charge neutrality per chemical species in a mixed system should be to consider two different unphysical Fermi levels for each sub-band, $E_{F, 0}^{A}$ and $E_{F, 0}^{B}$. In most cases, and in particular when $\mathrm{A}$ and $\mathrm{B}$ are late transition series elements with unfilled $d$-band, $E_{F, 0}^{A}$ and $E_{F, 0}^{B}$ are ordered in the same way as the corresponding barycentres $\varepsilon_{d, 0}^{A}$ and $\varepsilon_{d, 0}^{B}$. This is illustrated schematically in Fig. 18, and more precisely in the archetypal case of CoPt for which LDOS is represented for a 
L1 10 phase before any self-consistency. In such a situation the unphysical Fermi levels $E_{F, 0}^{i}$ fall into the upper part of the $d$ LDOS's. In view of the rather large order of magnitude of the density of states in this region, $E_{F, 0}^{i}$ weakly varies with $d$-band filling, and in particular significantly less than the barycentre, so that the epsilon sequence. In these conditions, the effect of self-consistency is obviously to merge the two unphysical Fermi levels into an intermediate single one, which requires opposite shifts of both $d$ levels and then reduces their difference $\delta_{d}$.

In view of these arguments, the only way to observe the reversal behaviour, i.e. an increase of $\delta_{\mathrm{d}}$ under self-consistency, should be to consider an alloy in which one of the two elements is a noble metal, with a full d-band, for which the unphysical Fermi level $E_{F, 0}^{i}$ falls into the low density $s p$ band, as schematically shown in Fig. 18, in which case it must be strongly shifted to ensure local charge neutrality. In such a situation, as can be guessed from Fig. 16a, except for $\mathrm{PdCu}$ system, the barycentre of the noble element sub-band is lower in energy than that of the transition element with unfilled $d$-band, which implies that, according to our sign convention, $\mathrm{A}$ is the transition element and $\mathrm{B}$ the noble one. In that case, according to Fig. 16a, one also satisfies $\delta_{n d}>0$, except for $\mathrm{B}=\mathrm{Au}$. Then the corresponding unphysical Fermi levels can be ordered in energy in the opposite way compared to the barycentres of the sub-bands, provided that the latter are not too distant (i.e., that $\delta_{d, O}$ is not too large), and that the narrow band is located below the larger one $\left(\delta_{n d}>0\right)$. In other words, one can expect a reversal behaviour as soon as $\delta_{d, O}$ and $\delta_{n d}$ have the same sign, provided that the former is not too large. This is indeed what occurs in the PtCu system, as can be seen in the same Fig. 18, for which the only way to achieve self-consistency is to move both levels in opposite directions, leading to an increase of $\delta_{d}$.

From this analysis one expects to observe different concentration dependence of the $d$ (and core) level lines under alloying in an $\mathrm{A}_{\mathrm{c}} \mathrm{B}_{1-\mathrm{c}}$ alloy. Indeed in the general case (decrease of $\delta_{d}$ ), one should observe a symmetrical behaviour of the curves associated to A and B where the level of each element decreases with increasing concentration $c$, whereas one expects a non symmetrical behaviour in the exceptional case (increase of $\delta_{d}$ ), the atomic level of the non noble metal remaining nearly constant on the overall concentration range close to its initial bulk value. This is indeed what occurs as illustrated in Fig. 19 by the variation of the $d$ levels corresponding to each constituent as a function of Pt concentration for the two alloys CoPt and PtCu. In the dilute limit, we show for the matrix only the $d$ levels of the atoms which are first neighbours of the impurity, the other ones keeping their bulk value. The overall behaviour is found in remarkable agreement with the evolution of core level shifts measured in XPS experiments [34] which are recalled in the insets, even though the calculated shifts are found larger than the experimental ones. Note that these curves also allow us to follow the variation with concentration of the diagonal disorder parameter $\delta_{d}$ with respect to the initial value calculated from pure metal data $\delta_{d, 0}$ which is found to be very weak in the general case $(\mathrm{CoPt})$ but larger in the exceptional one $(\mathrm{PtCu})$. Finally, the influence of concentration and of the off- 

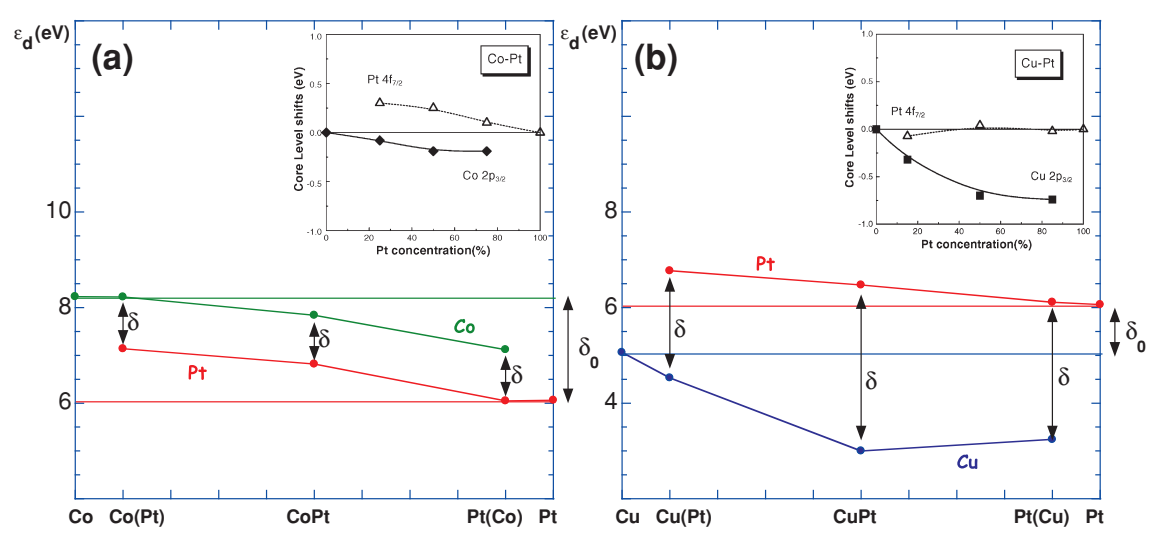

Fig. 19 Calculated shift of the atomic $d$ level as a function of the concentration in Pt atoms derived from TB calculations for $\mathrm{Co}$ and $\mathrm{Pt}$ atoms in $\mathrm{CoPt}$ alloy (a), and for $\mathrm{Cu}$ and $\mathrm{Pt}$ atoms in CuPt alloy (b) (from Ref. [32], copyright (2011) by The American Physical Society). The experimental absolute core level shifts taken from [34] are shown in the insets

diagonal disorder on the LDOS is illustrated in the Fig. 20 in the particular cases of the CoAu [15] and CoPt systems. As can be seen, it compares satisfactorily to that derived from DFT calculations.

\subsection{Rigid Lattice TBIM}

As previously stated for the pure metals, the total energy of the alloy, for a given configuration, cannot be described as a sum of pair interactions. Nevertheless, if one neglects (in a first time) the effect of off-diagonal disorder $\left(\delta_{n d}=0\right)$ the (small) part of the energy which depends explicitly on the configuration (and which is essential in ordering problems) can be written as a sum of effective pair interactions by developing the energy in a perturbative way with respect to the disordered state [35]:

$$
\begin{gathered}
E_{c o h}\left(\left\{p_{n}^{i}\right\}\right)=\bar{E}(c)+\frac{1}{2} \sum_{n, m, i, j} p_{n}^{i} p_{m}^{j} V_{n m}^{i j} \\
V_{n m}^{i j}=-\frac{\operatorname{Im}}{\pi} \int^{E_{F}} d E t_{n}^{i}(E) t_{m}^{j}(E) \sum_{\lambda \mu} \bar{G}_{n m}^{\lambda \mu}(E) \bar{G}_{m n}^{\mu \lambda}(E)
\end{gathered}
$$

in which the interatomic Green function: $\bar{G}_{n m}^{\lambda \mu}(E)=\langle n \lambda|\bar{G}(E)| m \mu\rangle$ is calculated using the CPA approximation developed in the previous section. Any energy balance which accounts for changes in the chemical configuration (mixing or ordering energies) will in fact involve the combination: 

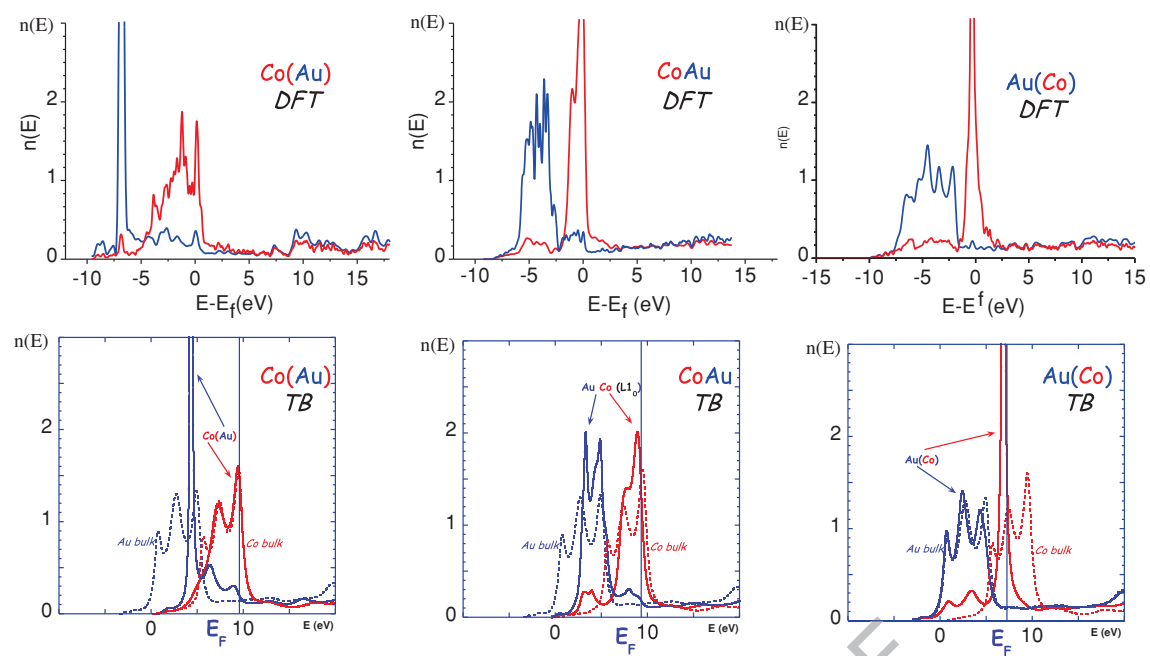

(a)
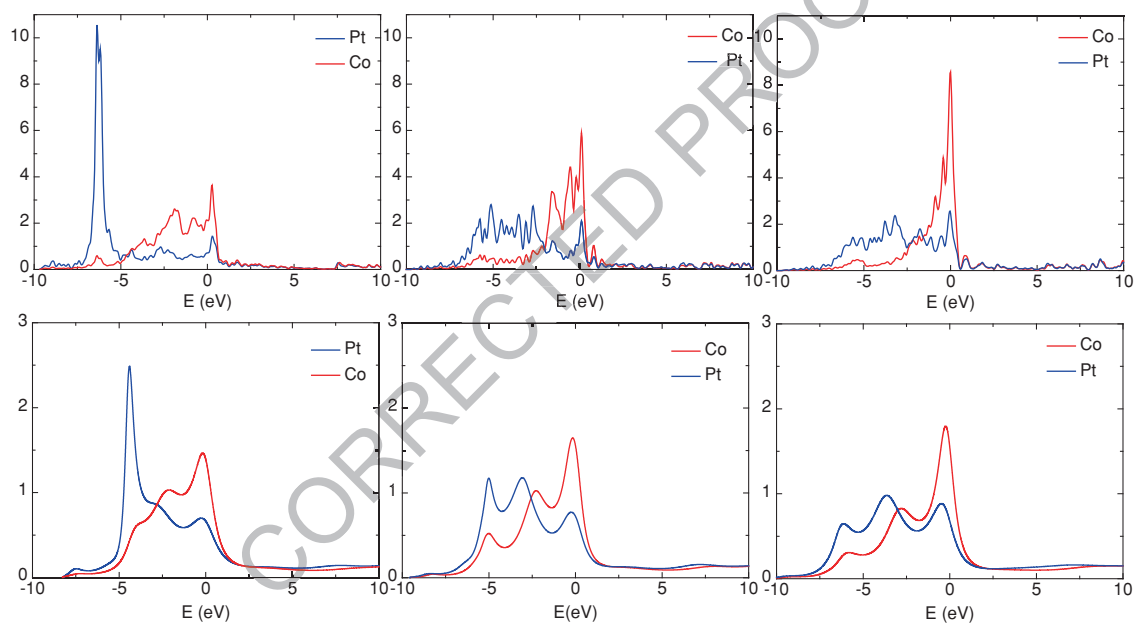

(b)

Fig. 20 Variation of the a Co-Au (from Ref. [15]) and b Co-Pt (courtesy of L. Zosiak) LDOS as a function of concentration from DFT and self-consistent TB calculations

$$
V_{n m}=\frac{\left(V_{n m}^{A A}+V_{n m}^{B B}-2 V_{n m}^{A B}\right)}{2}
$$

These effective pair interactions (EPI's) have been shown to decrease rapidly with the distance $(n-m)$ (for the fcc structure: $V_{1}>>V_{2}, V_{3}, V_{4}>>V_{5}, \ldots$ ). We will therefore limit ourselves to the first neighbour interactions which will be denoted $V=V_{1}$ in the following. In this framework the sign of $\mathrm{V}$ indicates the 
(a)

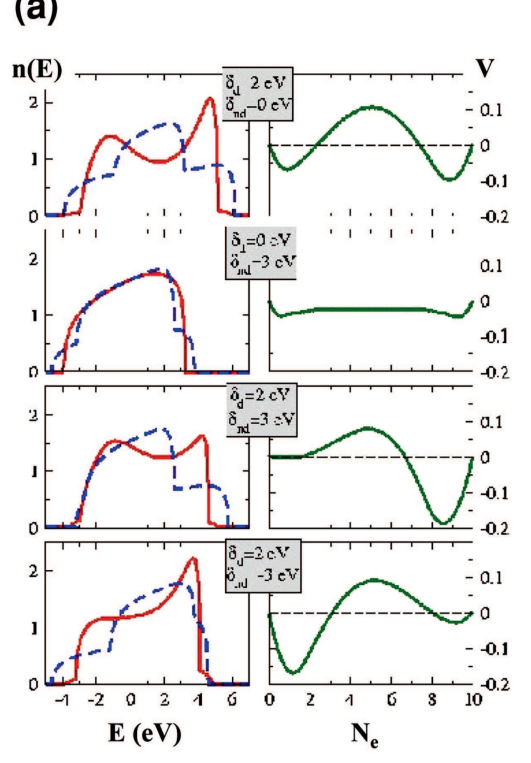

(b)

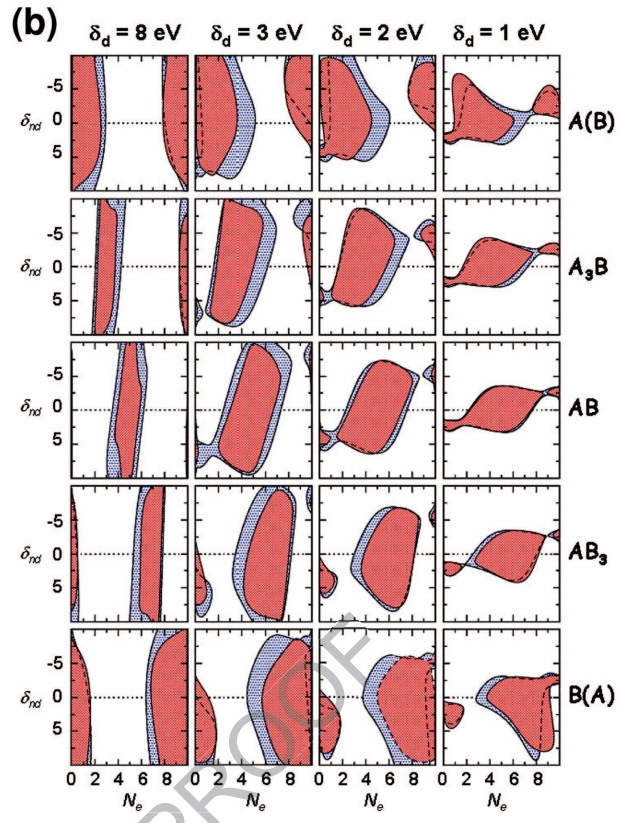

Fig. 21 (courtesy of J. Los): a Effects of diagonal $\left(\delta_{d}\right)$ and off-diagonal $\left(\delta_{n d}\right)$ disorder on the $d$ band LDOS, $n(E)$, (left-hand side) for the $L 1_{0}$ (solid line) and separated (dashed line) phases, and on the band-filling $\left(N_{e}\right)$ dependence of the effective pair interaction $V$ (right-hand side), with $\bar{W}=8 \mathrm{eV}$. (b) Ordering (shaded area) and demixing (white areas) domains in the parameter space spanned by $\delta_{n d}$ and $N_{e}$ for different values of $\delta_{d}$. From Ref. [36], copyright (2011) by The American Physical Society

tendency of the system to order $(V>0)$ or to phase-separate $(V<0)$. This sign depends (weakly) on bulk concentration (which could change the tendency to order or phase separate in a system in a few cases) and (strongly) on the average $d$ band filling $\bar{N}_{e}=c N_{e}^{A}+(1-c) N_{e}^{B}$ [35]. In practice, it is possible to calculate $V$ either directly from the above formula or indirectly from the expression of the formation energies (per atom) of some ordered phases $\left(\mathrm{E}_{\mathrm{form}}\left(\mathrm{L} 1_{0}\right)=-4 \mathrm{~V}\right.$, $E_{\text {form }}\left(L_{2}\right)=-3 \mathrm{~V}$ ), or from that of the solution energies $E_{\text {sol }}=-12 \mathrm{~V}$. Thus from the formation energy of the $\mathrm{L} 1_{0}$ phase for a realistic value of $\delta_{\mathrm{d}}$, calculated using continued fractions with two exact levels (fourth moment approximation: FMA) and a constant termination, one gets a typical variation of $\mathrm{V}$ shown in the Fig. 21a [36]. A comparison with the EPI's calculated with a larger set of exact moments [35] shows that truncating the continued fraction expansion to the second level is sufficient, which confirms the validity of the FMA [36]. As can be seen alloys with a nearly half filled band tend to order whereas those with nearly filled or empty bands tend to phase separate.

The main interest of such a simplified energetic model is that it can be efficiently implemented for lattice Monte-Carlo simulations in order to investigate ordering and segregation phenomena using appropriate values for the EPI's (see 
contribution by F. Ducastelle in Chap. 6). This approach was experimentally validated for a wide range of systems but some exceptions remained, among which, unfortunately, systems of high interest such as $\mathrm{CoPt}$, NiPt or $\mathrm{CuAu}$ which are predicted to phase separate although they are archetypal systems known to form ordered phases. It has then be argued that these discrepancies could be due to peculiar neglected effects, such as magnetism or spin-orbit coupling [10], without any definite conclusion up to now.

In addition to these serious drawbacks, a major problem of this Ising model is that it relies on a rigid lattice assumption which makes it completely unsuited to study the effects of relaxation by atomic displacements in the case of a strong sizemismatch between the constituents. In that case, a solution was to extend the semiempirical interatomic potentials previously developed for pure metal surfaces, among which the previously described Second Moment Approximation (SMA). However, in essence, this approximation only feels the off-diagonal disorder and not the diagonal disorder, since a second moment calculation, consisting of twohopping closed paths starting from an atom, does not involve the atomic levels of the neighbouring sites. The SMA is therefore not justified from the point of view of the Ising model based exclusively on the diagonal disorder effect.

The simplest extension that treats alike both diagonal and off-diagonal disorder effects is to use a LDOS based on a fourth moment approximation (FMA). Indeed this procedure allows first to revisit the TB Ising model, up to now limited to the diagonal disorder effect, by introducing off-diagonal disorder, and provides a well founded basis for a new generation of empirical potentials for alloys beyond SMA, based on the FMA, in particular for nanoalloys. Some typical LDOS's and corresponding EPI's for the $\mathrm{L1}_{0}$ phase as a function of the average $d$ band filling $N_{e}$ and different values of $\delta_{d}$ and $\delta_{n d}$ are shown in the same Fig. 21a. The case $\delta_{d}=0$ shows that the influence of off-diagonal disorder alone is to favour phase separation for any $d$ band filling. The behaviour of the EPI's in the two limit cases $\left(\delta_{d}=0\right.$ or $\left.\delta_{n d}=0\right)$ can be easily understood from simple qualitative arguments based on the respective band edges in the ordered and phase separated systems previously given. Finally, one sees that coupling both effects significantly modifies the previous curves by asymmetrising the $d$ band filling dependence, in an opposite way depending on the sign of $\delta_{n d}$, which in particular displaces the range of existence of ordering phases.

To generalize these curves, one can derive 3D maps from these EPI's, which, for a given concentration c, shows the tendency of a system to order or phase separate as a function of $\delta_{d}, \delta_{n d}$ (within the same physical ranges as in Fig. 16) and $N_{e}$. Sections of these 3D maps for different concentrations and selected values of $\delta_{d}$ are displayed in Fig. 21b. Each section shows the respective domains for the existence of ordered and separated phases as a function of $N_{e}$ (x-axis) and $\delta_{n d}$ (y-axis). As can be seen, the effect of off-diagonal disorder strongly changes the overall trends derived from calculations taking into account diagonal disorder only ( $\delta_{n d}=0$ in the maps). The most spectacular effect in this sense is probably the opening of ordering tendency domains, for reasonable values of the off-diagonal parameter, in the limits of small or large $d$ band fillings for which only phase 
separation was predicted before. The overall effect of concentration is to shift the ordering domains from larger to lower $d$ band filling from one dilute limit to the other. As a consequence, a given system can reverse its ordering tendency as a function of concentration, in particular for the largest values of $\delta_{d}$. However, let us keep in mind that the solution energy is very sensitive to atomic relaxations around the impurity which could change the map in the dilute case in presence of strong size-mismatch e.g., in the $\mathrm{CuAg}$ case [37].

There remains to see to what extent the ordering behaviour of real systems such as $\mathrm{CoPt}$, NiPt or $\mathrm{CuAu}$ which could not be explained by considering only diagonal disorder falls in the right place in the new domains. For this, we need to find the right point in the appropriate map of Fig. $21 \mathrm{~b}$, depending on the actual value of the parameters set $\left(\delta_{d}, \delta_{n d}, N_{e}\right), \delta_{d}$ (see Fig. 16) and $N_{e}$ being issued from the selfconsistent treatment upon $s p$ - $d$ hybridization. One then finds $(1,-2.9,8.35)$ for CoPt which then now falls into an ordering region as it should in the map of Fig. 21b without resorting to other effects such as magnetism or spin-orbit coupling. A similar agreement should be found in the map corresponding to $\delta_{d}=0.2$ for $\operatorname{NiPt}(0.2,-3.4,8.85)$. The new maps also allow us to find the right places for alloys made of two noble metals, which slipped through the previous description, and in particular to explain why two systems as close as $\mathrm{CuAg}(3.4,-0.6,9.85)$ and $\mathrm{CuAu}(1.6,-2.6,9.75)$ present two opposite behaviours, phase separation in the former case and ordering in the latter case.

\section{Alloy Surfaces and Clusters}

\subsection{Alloy Surfaces}

The LDOS at the surface of an alloy has to combine both bond breaking (as for pure metal surfaces) and alloying (as in bulk systems) effects. In particular, the self-consistency rule per species, site and orbital has to be applied for each inequivalent surface site depending on its occupation by A or B atoms. As shown in Fig. 22, for the CoAu system previously treated in the bulk (Fig. 20) but now in the particular configuration of a Co layer deposited on $\mathrm{Au}(111)$, this rule allows one to get LDOS in perfect agreement with DFT calculations.

At the difference of pure metal, the presence of the surface not only introduces atomic but also chemical rearrangements. Indeed, due to broken bonds, the equilibrium concentration at the surface has no reason to be the same as in the bulk, which leads to the phenomenon of surface segregation. The natural way for treating this problem is to extend to the case of surfaces the perturbation treatment of the energy (with respect to configuration fluctuations) previously developed for modelling the ordering processes in the bulk. This leads to the so-called TightBinding Ising Model (TBIM) that extends the Eq. (27) into [38]: 

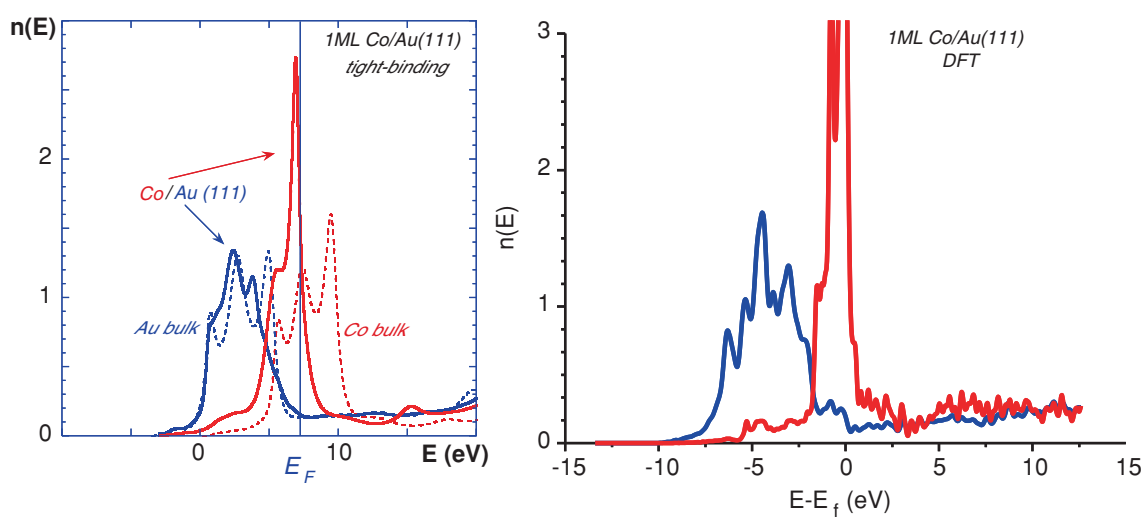

Fig. $22 \mathrm{Co} / \mathrm{Au}(111)$ LDOS from self-consistent TB (left) and DFT (right) calculations. From Ref. [15]

$$
H^{T B I M}\left(\left\{p_{n}^{i}\right\}\right)=\sum_{n, i} p_{n}^{i} h_{n}^{i}+\frac{1}{2} \sum_{n, m, i, j} p_{n}^{i} p_{m}^{j} V_{n m}^{i j}
$$

The main difference with the bulk case is the existence of sites which are no longer equivalent from the geometrical point of view, leading to a local on-site term:

$$
h_{n}^{i}=\frac{\operatorname{Im}}{\pi} \int^{E_{F}} d E \sum_{\lambda} \log \left[1-\left(\varepsilon^{i}-\sigma_{n}\right) \bar{G}_{n n}^{\lambda \lambda}(E)\right]
$$

In the simple case of a binary alloy $\left(p_{n}=p_{n}^{A}\right)$, one can determine the concentration profile $\{c p\}\left(c_{p}=<p_{n}>\right.$ for any site $n$ in the $p$ th plane parallel to the surface: $p=0$ ) as the one which minimises the free energy. Within mean-field approximation (see B. Legrand contribution in Chap. 7), the segregation energy, which accounts for the exchange of a A bulk atom with a B surface one, only involves the double differences: $\Delta h_{p}=\left(h_{p}^{A}-h_{p}^{B}\right)-\left(h_{\text {bulk }}^{A}-h_{\text {bulk }}^{B}\right)$. This local term $\Delta h_{p}$ is negligible in the bulk $(p>0)$ and almost identical to the difference in surface energies for $p=0\left(\Delta h_{0} \sim \tau^{A}-\tau^{B}\right)$ [38]. In fact, $\Delta h_{0}$ is the main driving force which leads to the segregation of the element with the lowest surface energy. The EPI's in Eq. (29) are also changed at the surface. More precisely, $V$ Eq. (28) is enhanced by a factor 1.5-2 with respect to its bulk value [38], at least in absence of size-mismatch.

Finally, let us note that, up to now, the derivation of TBIM has been performed on a rigid lattice, which is probably too crude in the case of large size mismatch between the constituents. However, there are two ways to introduce this effect. The first one is to add a third contribution to the segregation energy, $\Delta E_{p}^{s i z e}(c) \neq 0$ if $p=0$ (and 1 for open surfaces). $\Delta E_{0}^{\text {size }}(c)$ can be calculated in both dilute limits 
$(c \rightarrow 0,1)$ in the framework of SMA, by determining the four mixed A-B parameters in order that A and B only differ by their size [39]. This leads to a contribution which significantly differs from the one derived from elasticity theory since the latter leads in both limits to the segregation of the impurity, whatever its size. On the contrary, the SMA term is found strongly asymmetric, leading to a segregation of the impurity when it is the largest only (at least for close-packed surfaces). This comes from the anharmonicity of the potential which exhibits a strong asymmetry between tensile and compressive pressures. Size-mismatch can also strongly modify the EPI in the case of phase-separating systems such as $\mathrm{CuAg}$. Indeed, an SMA relaxation of a system containing two impurities shows that bond breaking can reverse the sign of $\mathrm{V}$ at the surface, leading to surface ordering in spite of bulk phase separation [37].

This so-called "three effects" rule (cohesive, alloying and size effects) proved to be quantitatively relevant for many different environments (flat or vicinal surfaces, grain boundaries, clusters) in alloys of transition metals with a chemical tendency to either phase separation $(\mathrm{CuAg})$ or to ordering with a low mixing energy, but not for systems with size-mismatch similar to $\mathrm{Cu}-\mathrm{Ag}$, but which exhibit a strong tendency to order $(\mathrm{CoPt})$. To elucidate the origin of this disagreement, a second approach has been proposed which couples these three effects (CTEM: Coupled Three Effects Model [40]), based on the systematic study of the permutation enthalpies in the bulk and at the surface as a function of the value of the mixed interaction parameter involved in the TBSMA potential. This allows one to explain both previous observations, disagreement for $\mathrm{CoPt}$ and agreement for $\mathrm{CuAg}$, as due to the variation of the EPI's at the surface and by the existence of coupling coefficients between the three effects. More specifically, if one indeed recovers that the surface EPIs are proportional to the bulk ones in the absence of significant size-mismatch, they are found to differ by an additive constant value in the presence of a strong size effect.

\subsection{As a Conclusion: Nanoalloys}

Determining the electronic structure of nanoalloys within TB approximation needs to combine the features of pure metal clusters and alloy surfaces. This means that one first has to extend the self-consistent neutrality rules per element, site and orbital by shifting the atomic levels differently for vertices, edges, facets depending on their occupation by A or B atoms to ensure the same orbital filling as in their respective bulks (coupling Figs. 15a, 16b and 19). The resulting LDOS per inequivalent surface sites should then also combine the features of those for pure clusters (Fig. 15b) and semi-infinite alloys (Fig. 22).

From the energetic point of view, the coupling between segregation and reconstruction [41] should be particularly important in bimetallic clusters, due to the effects of finite matter (the available quantity of segregant matter could be lower than the quantity of surface sites) and geometrical frustrations (coexistence 


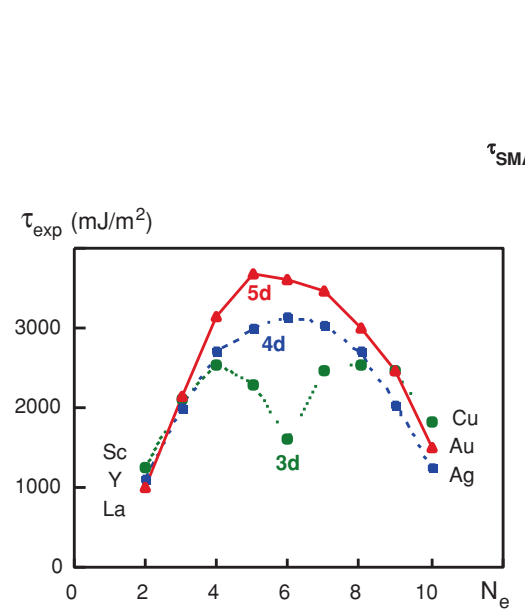

(a)

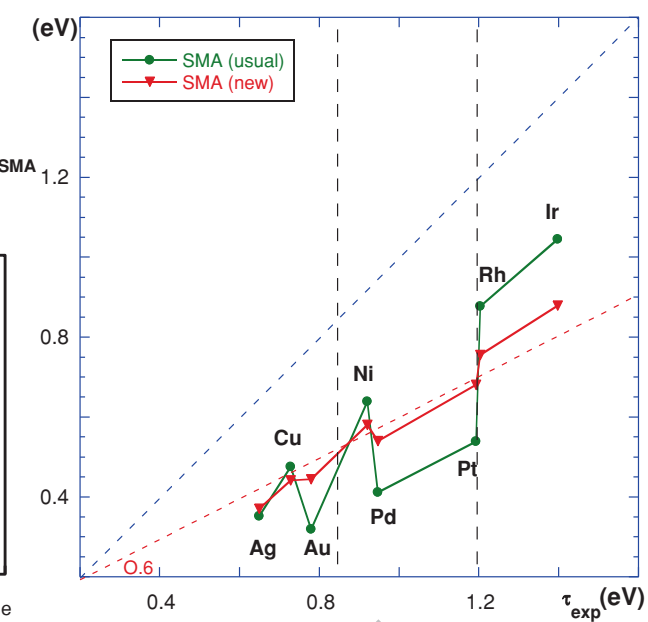

(b)

Fig. 23 a Experimental variation of the surface energy along the transition metal series. b SMA surface energy as a function of experimental one for usual parameters fitting only the cohesive energy (green line) and for parameters fitting also a value of surface energy of about 0.6 its actual value

of vertices, edges, facets with different orientations). In practice, this coupling between chemical and atomic structure is now too strong to allow us to separate them. A first attempt to achieve it is to mimic the case of alloy surfaces by combining TBIM/CTEM (segregation and ordering) and SMA (distance dependence of the interatomic potential) approaches, in order to be able to treat various morphologies (fcc polyhedra, bcc dodecahedra, icosahedra) and to study the competition or synergy between bulk ordering and surface segregation. This can be done in both directions, either using TBIM/CTEM on a rigid lattice with effective parameters (local field and EPI's) calculated by SMA relaxation procedure to account for size-mismatch, or reversely by using SMA potentials with parameters ensuring the TBIM/CTEM prescriptions by reproducing mixing energies and difference in surface energies between the constituents. This latter point is not obvious. Actually, as shown in Fig. 23, the usual SMA potential with parameters fitting bulk properties fails not only to reproduce the absolute values of these surface energies (which are found too small by a factor of two) but also, which is more important here, their variation from an element to the other. It is indeed impossible to fit simultaneously both cohesive energies and surface energies with this type of potential. A possible solution could be to accept this global lowering of the surface energies by introducing as an additional constraint in determining the SMA parameters that of reproducing $0.6 \tau^{\mathrm{i}}$ instead of $\tau^{\mathrm{i}}$. As can be seen in Fig. 23, this indeed allows recovering the good sequence from an element to the others. Such new SMA potentials are under development [42]. 
This coupled approach allowed to evidence the coupling between atomic relaxations and chemical arrangements, in two systems presenting opposite thermodynamics behaviours, $\mathrm{CuPd}$ [43] and $\mathrm{CuAg}$ [44] which respectively tend to order and to phase separate. Thus in the CuPd case, for small sizes, if the usual sequence of relative stabilities (icosahedron, fcc, and -well above- bcc dodecahedron) was recovered in the disordered state, chemical ordering at low temperature leads to a spectacular reversal in which the bcc structure is stabilized with respect to fcc by chemistry, the icosahedron being destabilized by chemical order. Moreover, a surface induced disorder is observed with respect to inner sites. On the other hand, in the $\mathrm{CuAg}$ case, one finds that the segregation hierarchy based on broken-bond arguments (preferential segregation to the vertices, less to edges, and least to facets) is not at all universal and that the segregation driving forces for cuboctahedral and icosahedral nanoalloys may differ, being similar for the vertex and edge sites, but not for the sites of the triangular facets due to dilations of orthoradial distances in the icosahedral structure.

The alternative solution to this mixed approach is to directly couple the chemical and atomic requirements by giving up SMA/TBIM in favour of FMA potentials. Indeed, the previous results on ordering trends in bulk alloys (Fig. 21) not only allow to revisit the TB Ising model by accounting for both diagonal and off-diagonal disorder effects, but also provide a well founded basis for a future extensive use in nanoalloys of FMA interatomic potentials, up to now limited to covalent materials [45] or pure metals [46]. Here also some work is currently done for the archetypal $\mathrm{CoPt}$ and $\mathrm{CuAg}$ systems.

\section{References}

1. Kohn, W., Becke, A.D., Parr, R.G.: Density functional theory of electronic structure. J. Phys. Chem. 100, 12974-12980 (1996) (and references therein)

2. Ashcroft, N.W.: The Fermi surface of aluminium. Philos. Mag. 8, 2055-2083 (1963)

3. Car, R., Parrinello, M.: Unified approach for molecular dynamics and density-functional theory. Phys. Rev. Lett. 55, 2471-2474 (1992)

4. Friedel, J.: Physics of Metals, vol. 1, Cambridge University Press, Cambridge (1978)

5. Ducastelle, F.: Structure électronique des métaux de transition et de leurs alliages. Thèse Orsay (1972)

6. Lambin, P., Gaspard, J.P.: Continued-fraction technique for tight-binding systems: a generalized-moments method. Phys. Rev. B 26, 4356-4368 (1982)

7. Haydock, R., Heine, V., Kelly, M.J.: Electronic structure based on local atomic environment for tight-binding bands. J. Phys. C 5, 2845-2858 (1972); C 8, 2591-2605 (1975)

8. Turchi, P., Ducastelle, F., Tréglia, G.: Band gaps and asymptotic behaviour of continued fraction coefficients. J. Phys. C 15, 2891-2924 (1982)

9. Jaafar, A., Goyhenex, C., Tréglia, G.: Role of sp-d hybridization in the formation of stacking defects at metal surfaces. Surf. Sci. 602, 2681-2688 (2008)

10. Ducastelle, F.: Order and Phase Stability in Alloys. North-Holland, Amsterdam (1991)

11. Turchi, P.: Structure électronique et stabilité des alliages de métaux de transition: effets de structure cristalline et d'ordre configurationnel. Thèse Paris (1984)

12. Desjonquères, M.C., Spanjaard D.: Concepts in Surface Physics. Springer, Berlin (1995) 
13. Spanjaard, D., Guillot, C., Desjonquères, M.C., Tréglia, G., Lecante, J.: Surface core level spectroscopy of transition metals: a new tool for the determination of their surface structure. Surf. Sci. Rep. 5, 1-85 (1985)

14. Sawaya, S., Goniakowski, J., Mottet, C., Saúl, A., Tréglia, G.: Charge redistribution at Pd surfaces: ab initio grounds for tight-binding interatomic potentials. Phys. Rev. B 56, 12161-12166 (1997)

15. Jaafar, A., Goyhenex, C., Tréglia, G.: Rules for tight-binding calculations in bimetallic compounds based on density functional theory: the case of CoAu. J. Phys. Condens. Matter. 22, 505503 (2010)

16. Rosato, V., Guillopé, M., Legrand, B.: Thermodynamical and structural properties of fcc transition metals using a simple tight-binding model. Philos. Mag. A 59, 321-336 (1989)

17. Spanjaard, D., Desjonquères, M.C.: Universal features of bonding in metals. Phys. Rev. B 30, 4822-4827 (1984)

18. Foiles, S.M., Baskes, M.I., Daw, M.S.: Embedded atom method functions for the fcc metals $\mathrm{Cu}, \mathrm{Ag}, \mathrm{Au}, \mathrm{Ni}, \mathrm{Pd}, \mathrm{Pt}$ and their alloys. Phys. Rev. B 33, 7983-7991 (1986)

19. Garofalo, M., Tosatti, E., Ercolessi, F.: Structure, energetics, and low temperature behaviour of the $\mathrm{Au}(110)$ reconstructed surface. Surf. Sci. 188, 321-326 (1987)

20. Guillopé, M., Legrand, B.: (110) surface stability in noble metals. Surf. Sci. 215, 577-595 (1989)

21. Legrand, B., Tréglia, G., Desjonquères, M.C., Spanjaard, D.: A "quenched molecular dynamics" approach to the atomic stability of the (100) face of bcc transition metals. J. Phys. C 19, 4463-4472 (1986)

22. Mottet,C.: Étude par simulation numérique d'agrégats libres mono- et bi-métalliques. Thèse, Université Aix-Marseille II (1997)

23. Mottet, C., Tréglia, G., Legrand, B.: Electronic structure of Pd clusters in the tight-binding approximation: influence of spd-hybridization. Surf. Sci. 352-354, 675-679 (1996)

24. Hammer, B., Morikawa, Y., Norskov, J.K.: CO Chemisorption at metal surfaces and overlayers. Phys. Rev. Lett. 76, 2141-2144 (1996)

25. Mottet, C., Tréglia, G., Legrand, B.: New magic numbers in metallic clusters: an unexpected metal dependence. Surf. Sci. 383, L719-L727 (1997)

26. Wang, L.L., Johnson, D.D.: Density functional study of structural trends for late-transitionmetal 13-atom clusters. Phys. Rev. B 75, 235405 (2007)

27. Papaconstantopoulos, D.A.: Handbook of Electronic Structure of Elemental Solids. Plenum, New York (1986)

28. Velicky, B., Kirkpatrick, S., Ehrenreich, H.: Single-site approximations in the electronic theory of simple binary alloys. Phys. Rev. B 175, 747-766 (1968)

29. Bieber, A., Ducastelle, F., Gautier, F., Tréglia, G., Turchi, P.: Electronic structure and relative stabilities of $\mathrm{L}_{12}$ and $\mathrm{DO}_{22}$ ordered structures occurring in transition metal alloys. Solid State Comm. 45, 585-590 (1983)

30. Kudrnovsky, J., Bose, S.K., Andersen, O.K.: Comparative study of the electronic structure of ordered, partially ordered and disordered phases of the $\mathrm{Cu}_{3} \mathrm{Au}$ alloy. Phys. Rev. B 43, 4613-4621 (1991)

31. Olovsson, W., Göransson, C., Pourovski, L.V., Johansson, B., Abrikosov, I.A.: Core-level shifts in fcc random alloys: a first-principles approach. Phys. Rev. B 72, 064203 (2005)

32. Goyhenex, C., Tréglia, G.: Unified picture of d band and core level shifts in transition metal alloys. Phys. Rev. B 83, 075101 (2011)

33. Tréglia, G., Ducastelle, F., Gautier, F.: Generalised perturbation theory in disordered transition metal alloys: application to the self-consistent calculation of ordering energies. J. Phys. F 8, 1437-1456 (1978)

34. Lee, Y.-S., Lim, K.-Y., Chung, Y.-D., Wang, C.-N., Jeon, Y.: XPS core-level shifts and XANES studies of Cu-Pt and Co-Pt alloys. Surf. Interface Anal. 30, 475-478 (2000)

35. Bieber, A., Gautier, F., Tréglia, G., Ducastelle, F.: Electronic structure, pairwise interactions and ordering energies in binary fcc transition metal alloys. Solid State Comm. 39, 149-153 (1981) 
36. Los, J., Mottet, C., Tréglia, G., Goyhenex, C.: Ordering trends in transition metal alloys from tight-binding electronic structure calculations. Phys. Rev. B 84, 180202(R) (2011)

37. Meunier, I., Tréglia, G., Legrand, B.: Surface-induced ordering in phase separation systems: Influence of concentration and orientation. Surf. Sci. 441, 225-239 (1999)

38. Tréglia, G., Legrand, B., Ducastelle, F.: Segregation and ordering at surfaces of transition metal alloys: the tight-binding ising model. Europhys. Lett. 7, 575-580 (1988)

39. Tréglia, G., Legrand, B.: Surface-sandwich segregation in PtNi and AgNi alloys: two different physical origins for the same phenomenon. Phys. Rev. B 35, 4338-4344 (1987)

40. Creuze, J., Braems, I., Berthier, F., Mottet, C., Tréglia, G., Legrand, B.: Model of surface segregation driving forces and their coupling. Phys. Rev. B 78, 075413 (2008)

41. Tréglia, G., Legrand, B., Ducastelle, F., Saúl, A., Gallis, C., Meunier, I., Mottet, C., Senhaji, A.: Alloy surfaces: Segregation, reconstruction and phase transitions. Comput. Mat. Sci. 15, 196-235 (1999)

42. Goyhenex, C.: Revised tight-binding second moment potential for transition metal surfaces. Surf. Sci. 606(3-4), 325-328 (2012)

43. Mottet, C., Tréglia, G., Legrand, B.: Theoretical investigation of chemical and morphological ordering in $\mathrm{Pd}_{\mathrm{c}} \mathrm{Cu}_{1-\mathrm{c}}$ clusters. Phys. Rev. B 66, 045413 (2002)

44. Moreno, V., Creuze, J., Berthier, F., Mottet, C., Tréglia, G., Legrand, B.: Site segregation in size-mismatched nanoalloys: application to $\mathrm{Cu}-\mathrm{Ag}$. Surf. Sci. 600, 5011-5020 (2006)

45. Amara, H., Bichara, C., Ducastelle, F.: Understanding the nucleation mechanisms of carbon nanotubes in catalytic chemical vapor deposition. Phys. Rey. Lett. 100, 056105 (2008)

46. Los, J.H., Pellenq, J.M.: Determination of the bulk melting temperature of nickel using Monte Carlo simulations: inaccuracy of extrapolation from cluster melting temperatures. Phys. Rev. B 81, 064112 (2010) 